\title{
A taxonomically harmonized and temporally standardized fossil pollen dataset from Siberia covering the last $40 \mathrm{kyr}$
}

\author{
Xianyong Cao ${ }^{1, \mathrm{a}}$, Fang Tian ${ }^{1}$, Andrei Andreev ${ }^{1,2}$, Patricia M. Anderson ${ }^{3}$, Anatoly V. Lozhkin ${ }^{4}$, \\ Elena Bezrukova $^{5,6}$, Jian Ni $^{7}$, Natalia Rudaya ${ }^{1,6}$, Astrid Stobbe ${ }^{8}$, Mareike Wieczorek ${ }^{1}$, and \\ Ulrike Herzschuh ${ }^{1,9,10}$ \\ ${ }^{1}$ Alfred Wegener Institute for Polar and Marine Research, Research Unit Potsdam, \\ Telegrafenberg A43, 14473 Potsdam, Germany \\ ${ }^{2}$ Institute of Geology and Petroleum Technologies, Kazan Federal University, \\ Kremlevskaya 18, 420008 Kazan, Russia \\ ${ }^{3}$ Earth and Space Sciences and Quaternary Research Center, \\ University of Washington, Seattle, WA 98185, USA \\ ${ }^{4}$ North-East Interdisciplinary Science Research Institute, Far East Branch \\ Russian Academy of Sciences, 685000 Magadan, Russia \\ ${ }^{5}$ Vinogradov Institute of Geochemistry, Siberian Branch, Russian Academy of Sciences, \\ ul. Favorskogo 1a, 664033 Irkutsk, Russia \\ ${ }^{6}$ Institute of Archeology and Ethnography, Siberian Branch, Russian Academy of Sciences, \\ pr. Akad. Lavrentieva 17, 630090 Novosibirsk, Russia \\ ${ }^{7}$ College of Chemistry and Life Sciences, Zhejiang Normal University, \\ Yingbin Road 688, 321004 Jinhua, China \\ ${ }^{8}$ Goethe University, Norbert-Wollheim-Platz 1, 60629 Frankfurt am Main, Germany \\ ${ }^{9}$ Institute of Environmental Sciences and Geography, University of Potsdam, \\ Karl-Liebknecht-Str. 24, 14476 Potsdam, Germany \\ ${ }^{10}$ Institute of Biochemistry and Biology, University of Potsdam, \\ Karl-Liebknecht-Str. 24, 14476 Potsdam, Germany \\ a present address: Key Laboratory of Alpine Ecology, CAS Center for Excellence in Tibetan Plateau Earth \\ Sciences, Institute of Tibetan Plateau Research, Chinese Academy of Sciences, \\ 100101 Beijing, China \\ Correspondence: Ulrike Herzschuh (ulrike.herzschuh@awi.de) and Xianyong Cao (xcao@itpcas.ac.cn)
}

Received: 12 January 2019 - Discussion started: 28 May 2019

Revised: 12 November 2019 - Accepted: 9 December 2019 - Published: 22 January 2020

\begin{abstract}
Pollen records from Siberia are mostly absent in global or Northern Hemisphere synthesis works. Here we present a taxonomically harmonized and temporally standardized pollen dataset that was synthesized using 173 palynological records from Siberia and adjacent areas (northeastern Asia, 42-75 ${ }^{\circ} \mathrm{N}, 50-180^{\circ} \mathrm{E}$ ). Pollen data were taxonomically harmonized, i.e. the original 437 taxa were assigned to 106 combined pollen taxa. Age-depth models for all records were revised by applying a constant Bayesian age-depth modelling routine. The pollen dataset is available as count data and percentage data in a table format (taxa vs. samples), with age information for each sample. The dataset has relatively few sites covering the last glacial period between 40 and $11.5 \mathrm{ka}$ (calibrated thousands of years before $1950 \mathrm{CE}$ ) particularly from the central and western part of the study area. In the Holocene period, the dataset has many sites from most of the area, with the exception of the central part of Siberia. Of the 173 pollen records, $81 \%$ of pollen counts were downloaded from open databases (GPD, EPD, PANGAEA) and $10 \%$ were contributions by the original data gatherers, while a few were digitized
\end{abstract}


from publications. Most of the pollen records originate from peatlands (48\%) and lake sediments (33\%). Most of the records $(83 \%)$ have $\geq 3$ dates, allowing the establishment of reliable chronologies. The dataset can be used for various purposes, including pollen data mapping (example maps for Larix at selected time slices are shown) as well as quantitative climate and vegetation reconstructions. The datasets for pollen counts and pollen percentages are available at https://doi.org/10.1594/PANGAEA.898616 (Cao et al., 2019a), also including the site information, data source, original publication, dating data, and the plant functional type for each pollen taxa.

\section{Introduction}

Continental or sub-continental pollen databases are essential for spatial reconstructions of former climates and past vegetation patterns of the terrestrial biosphere and in interpreting their driving forces (Cao et al., 2013); they also provide data for use in palaeodata-model comparisons at a continental scale (Gaillard et al., 2010; Trondman et al., 2015). Continental pollen databases from North America, Europe, Africa, and Latin America have been successfully established (Gajewski, 2008), and a fossil pollen dataset has been established for the eastern part of continental Asia (including China, Mongolia, southern Siberia, and parts of Central Asia; Cao et al., 2013). These datasets have been used to infer the locations of glacial refugia and migrational pathways by pollen mapping (e.g. Magri, 2008; Cao et al., 2015) and to reconstruct biome or land cover (e.g. Ni et al., 2014; Trondman et al., 2015; Tian et al., 2016) and climates at broad spatial scales (e.g. Mauri et al., 2015; Marsicek et al., 2018).

Pollen records from Siberia have rather seldomly been included in global, Northern Hemisphere, or synthesis works (Sanchez Goñi et al., 2017; Marsicek et al., 2018), probably because (1) few records are available in open databases or (2) available data are not taxonomically harmonized and lack reliable chronologies. Binney et al. (2017) established a pollen dataset together with a plant macrofossil dataset for northern Eurasia (excluding East Asia; the dataset has not been made accessible yet), but the chronologies were not standardized and the pollen data restricted to 1000-year time slices. In addition, a few works that make use of Siberian fossil pollen data either present biome reconstructions (Binney et al., 2017; Tian et al., 2018), which do not require taxonomic harmonization of the data, or restrict the analyses to selected times slices such as 18, 6, and $0 \mathrm{ka}$ (Tarasov et al., 1998, 2000; Bigelow et al., 2003).

Here we provide a new taxonomically harmonized and temporally standardized fossil pollen dataset for Siberia and adjacent areas.

\section{Dataset description}

\subsection{Data sources}

We obtained 173 late Quaternary fossil pollen records (generally since $40 \mathrm{ka}$ ) from Siberia and surrounding areas (42$75^{\circ} \mathrm{N}, 50-180^{\circ} \mathrm{E}$ ) from database sources and/or contributors or by digitizing published pollen diagrams (Appendix A; this table is available in PANGAEA). A total of 102 raw pollen count records were downloaded from the Global Pollen Database (GPD; http://www.ncdc.noaa.gov/paleo/gpd.html, last access: August 2010); 18 pollen count records were downloaded from the European Pollen Database (EPD; http: //www.europeanpollendatabase.net, last access: July 2016); 20 pollen records (16 sites have pollen count data, others with pollen percentages) were collected from the PANGAEA website (Data Publisher for Earth and Environmental Science, which also includes most pollen records found in GPD and EPD; https://www.pangaea.de, last access: July 2016); raw pollen count data of 17 sites were contributed directly by the data gatherers; and pollen percentages for the remaining 16 sites were digitized from the published pollen diagrams.

\subsection{Data processing}

Pollen standardization follows Cao et al. (2013), including homogenization of taxonomy at family or genus level generally (437 pollen names were combined into 106 taxa; Appendix B; this table is available in PANGAEA) and recalculation of pollen percentages on the basis of the total number of terrestrial pollen grains. To obtain comparable chronologies, age-depth models for these pollen records were re-established using Bayesian age-depth modelling with the IntCal09 radiocarbon calibration curve ("Bacon" software; Blaauw and Christen, 2011). We set up a gamma distribution accumulation rate with a shape parameter equal to 2, a beta distribution with a "strength" of 20 for all records for the accumulation variability, a mean "memory" of 0.1 for lake sediments, and a high memory of 0.7 for peat and other sediment types (following Blaauw and Christen, 2011). For the 20 pollen records without raw pollen counts, we set the terrestrial pollen sum based on the descriptions given in the original publications. Approximate values or ranges were provided for 16 records, e.g. more than 600 for the pollen record from Chernaya Gorka palsa and between 452 and 494 grains for Two-Yurts Lake, these pollen sums are assigned at 


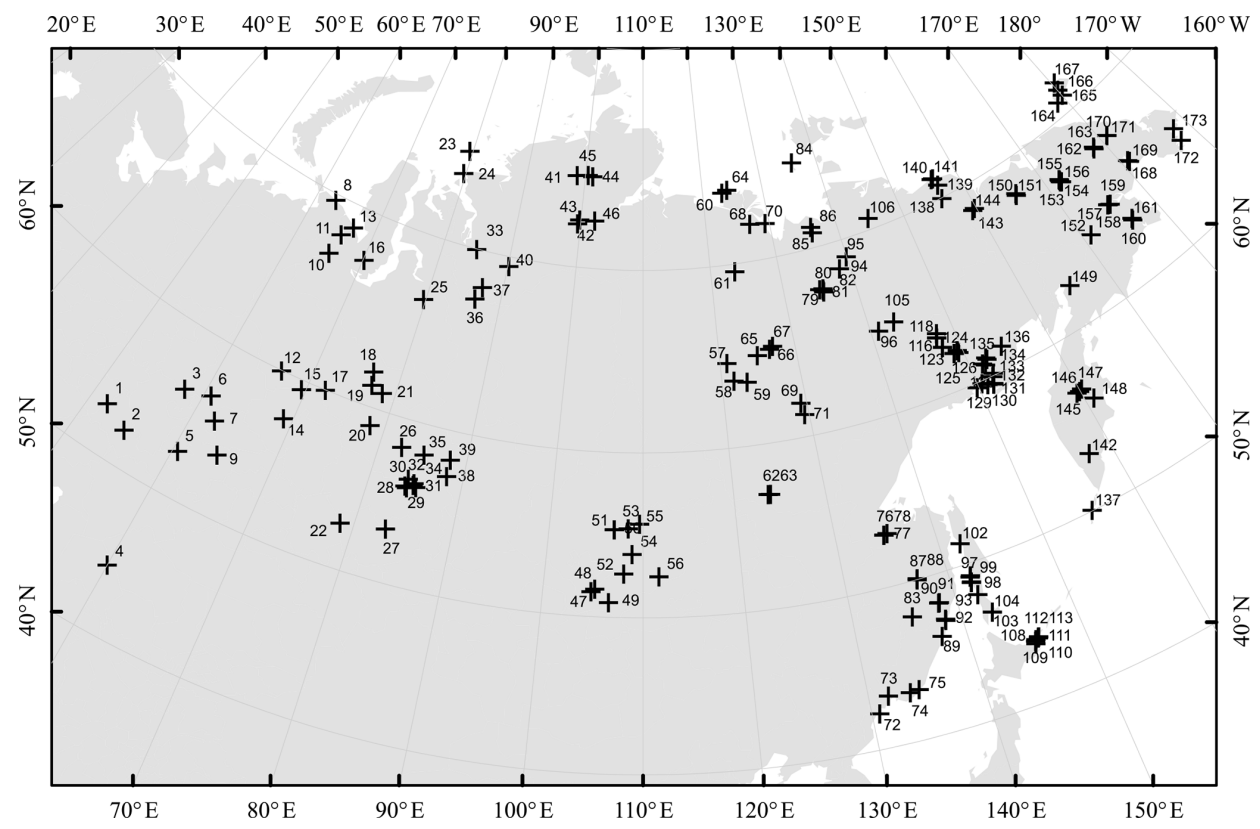

Figure 1. Spatial distribution of fossil pollen records (+) in the study area. The number of each site is used as its ID in Table A1.

600 and 470, respectively. A pollen sum of 400 is assigned for the other four records because no information was provided in the publications.. The "pollen counts" were then back-calculated using the pollen percentages and pollen sum. Finally, the pollen datasets are available with both count data and percentage data in table format in Excel software (taxa vs. samples), with age and location information for each sample.

\subsection{Data quality}

The Siberia pollen dataset includes pollen count data and percentages from 173 pollen sampling sites (Fig. 1). Sites are distributed reasonably evenly in eastern and western Siberia, but geographic gaps still exist in central Siberia $\left(55-70^{\circ} \mathrm{N}\right.$, $90-120^{\circ} \mathrm{E}$ ), where no published pollen records exist.

The dataset includes 83 pollen records from peat sediments, 57 records from lake sediments, 23 from fluvial sediments, 6 from coastal or marine sediments, 3 from palaeosol profiles, and 1 from palsa sediment (Appendix A). The peat and lake sediments generally have reliable chronologies and high sampling resolutions of the pollen records. About $83 \%$ of the pollen records have $\geq 3$ dates ( $~ 57 \%$ have $\geq 5$ dates); $73 \%$ of the pollen records have sampling resolutions of $<$ 500 years per sample and only $14 \%$ sites have $>1000$ years per sample (Appendix A).

Within this dataset, $91 \%$ of the pollen records (157 sites) have raw pollen count data or percentages with complete pollen assemblages (Appendix A). Although there might be some rare pollen taxa excluded from the published pollen diagrams (16 sites) that were digitized, these pollen taxa are likely of minor importance within the pollen assemblages.
In addition, during digitizing we ensured that the sum of pollen percentages for each pollen assemblage was within $100 \pm 10 \%$, to minimize artificially introduced errors.

The pollen records were counted by different scientists that gave different pollen names to the same pollen types requiring taxonomic homogenization (from 437 original taxa to 106 combined taxa). However, this reduces the taxonomic resolution of the dataset. In cases where homogenization would have resulted in grouping pollen taxa with different growth forms (herb, shrub, or tree) together, we keep the taxa separately even though not all analysts separated them (for instance, Betula pollen is separated into Betula_shrub, Betula_tree and Betula_undiff). We also append the original pollen names to the dataset to ensure feasibility of future studies on various topics using these data.

The chronologies of most pollen records are based on a reasonable number of dates (mostly ${ }^{14} \mathrm{C}$; at least 3 dates per record). However, we also included pollen records from under-represented areas or periods that do not meet this criterion. Furthermore, most of the pollen records cover only part of the last $40 \mathrm{kyr}$, and comparatively few pollen records cover (parts of) the last glacial (i.e. $>11 \mathrm{ka}$ ). We interpolated pollen abundances at 16 key time slices $(40,25,15$, $13,11,10,9,8,7,6,5,4,3,1.5$, and $0.5 \mathrm{ka}$ ) using the interp.dataset function in the $\mathrm{R}$ package rioja (Juggins, 2012) to produce pollen presence-absence maps for Larix as an example of the distribution of available sites at these $16 \mathrm{key}$ time slices (Fig. 2). We also present boxplots for 14 major pollen taxa from all available sites at the 16 key time slices (Fig. 3), which illustrates the general temporal patterns. 

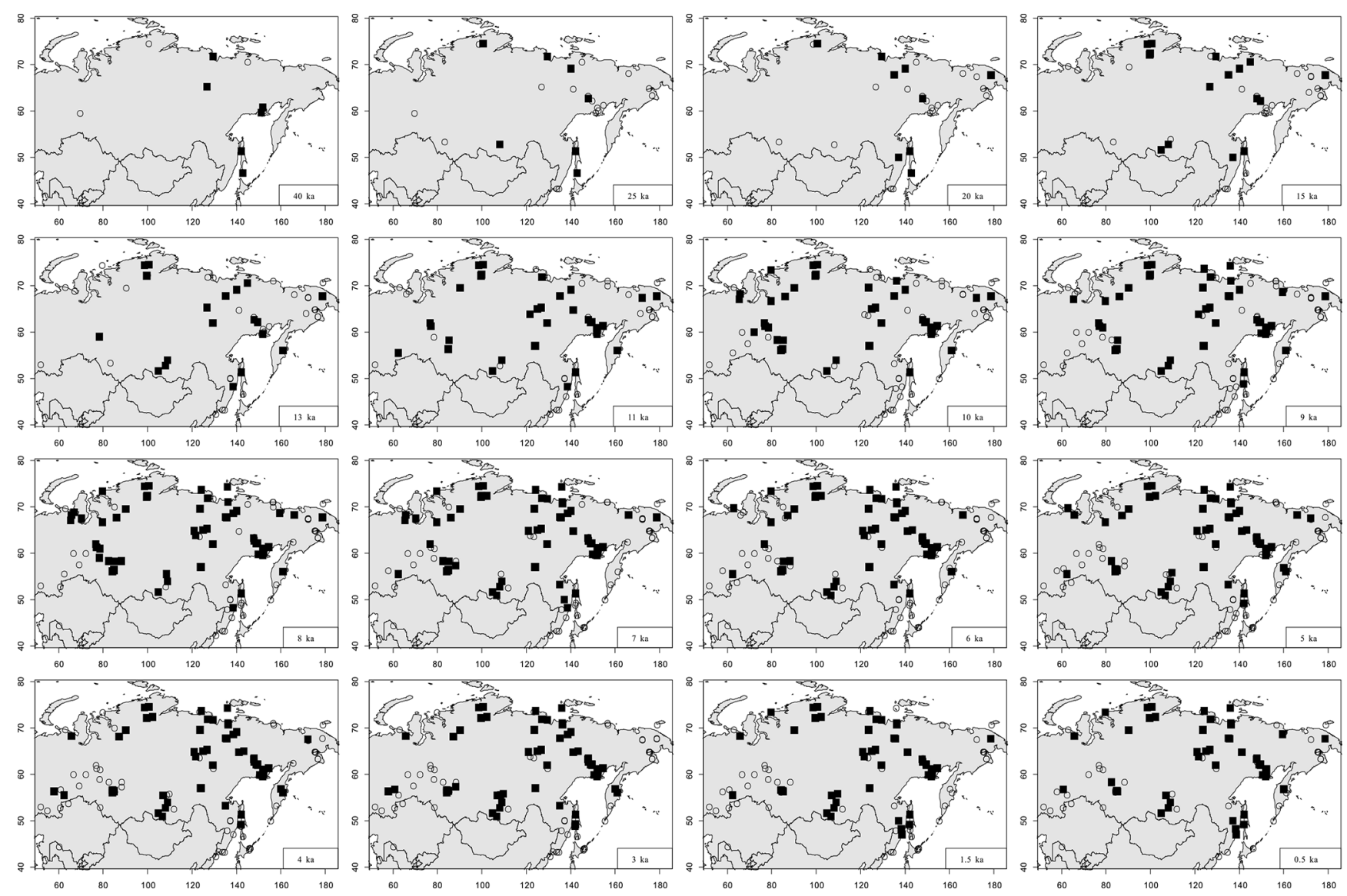

Figure 2. Pollen-inferred presence-absence maps for Larix at key time slices. Black squares indicate presence while empty circles indicate absence.

\section{Potential use of the Siberian fossil pollen dataset}

Fossil pollen data mapping can be used to reveal broadscale spatial distributions over time, as Cao et al. (2015) demonstrate. In this paper, we present presence-absence maps for Larix as an example (Fig. 2). Larix has extremely low pollen productivity (e.g. Niemeyer et al., 2015) that causes the under-representation of Larix pollen compared to its cover in the pollen source vegetation (Lisitsyna et al., 2011). Accordingly, Larix pollen is accepted as an indicator of the presence of Larix locally (e.g. Lisitsyna et al., 2011). The pollen presence-absence maps for Larix (Fig. 2) show a wide geographical range over the last 40000 years, even during the Last Glacial Maximum, when there was very likely a relatively low density of larch. Our results generally confirm the distribution revealed by Larix macrofossil analysis (Binney et al., 2009). The Larix distribution changes revealed by our pollen dataset exemplify the usability of the dataset for vegetation reconstruction.

The Siberian fossil pollen dataset has already been used for biome reconstruction (Tian et al., 2018), although an integration of this dataset into global or Northern Hemispherewide biomization research is still pending.
Pollen percentages in pollen assemblages do not directly reflect species abundance in the vegetation community because of different pollen productivity. Therefore, quantitative vegetation composition is modelled using pollen productivity estimates (e.g. Sugita et al., 2010; Trondman et al., 2015). Our pollen dataset was recently used to reconstruct plant cover quantitatively using the REVEALS model to describe the compositional changes in space and time, which is more reliable than using pollen percentages directly (Cao et al., 2019b).

Modern pollen data have been published from many sites in Siberia (e.g. Tarasov et al., 2007, 2011; Müller et al., 2010; Klemm et al., 2015). These modern pollen datasets can be used to investigate modern pollen-climate relationships, and these modern relationships can be used to make quantitative climate reconstructions, as has been done previously (e.g. Marsicek et al., 2018).

\section{Data availability}

Five datasets including overview and reference (site information), dating data, plant functional type for each pollen taxa, 

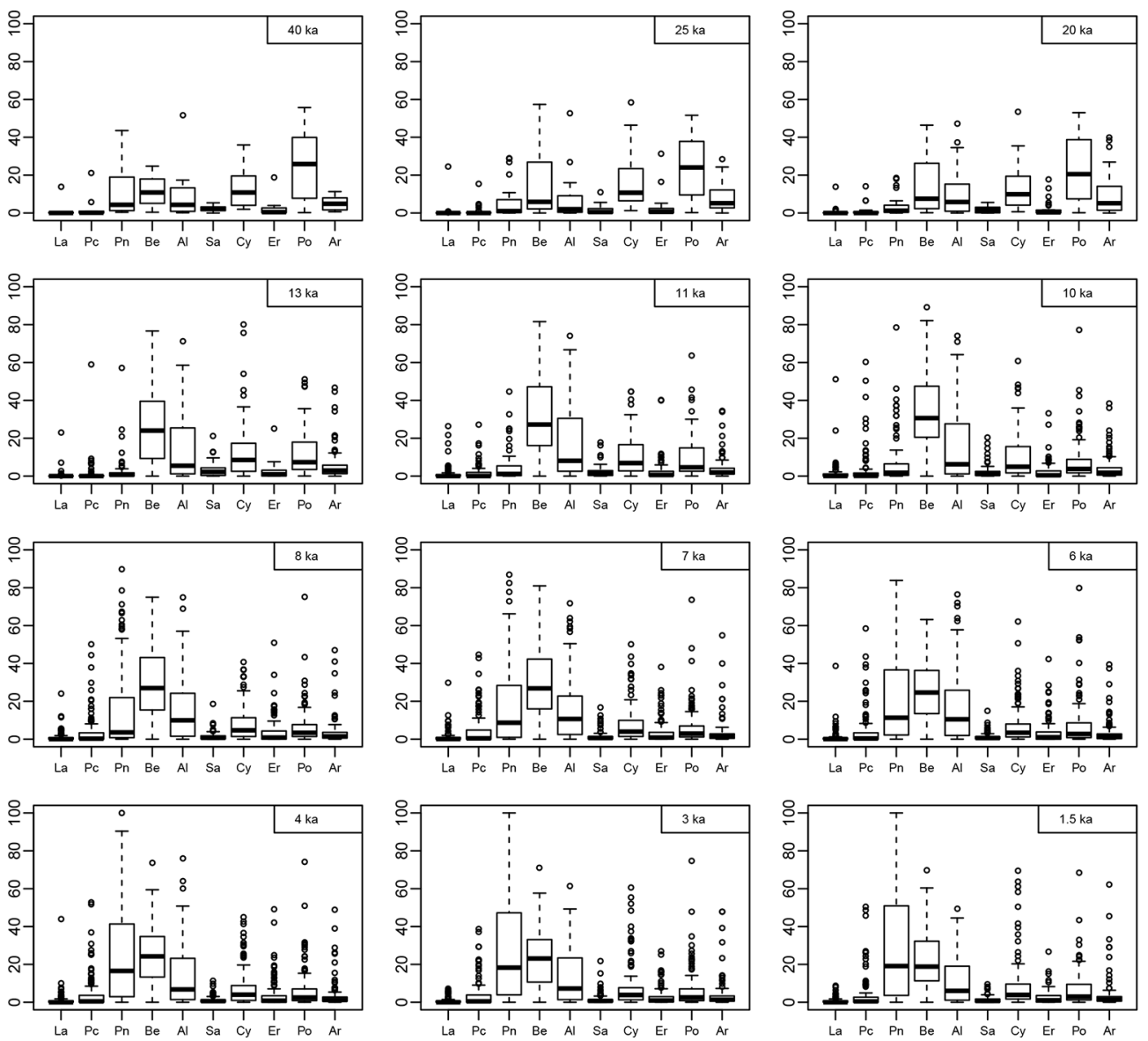

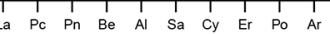
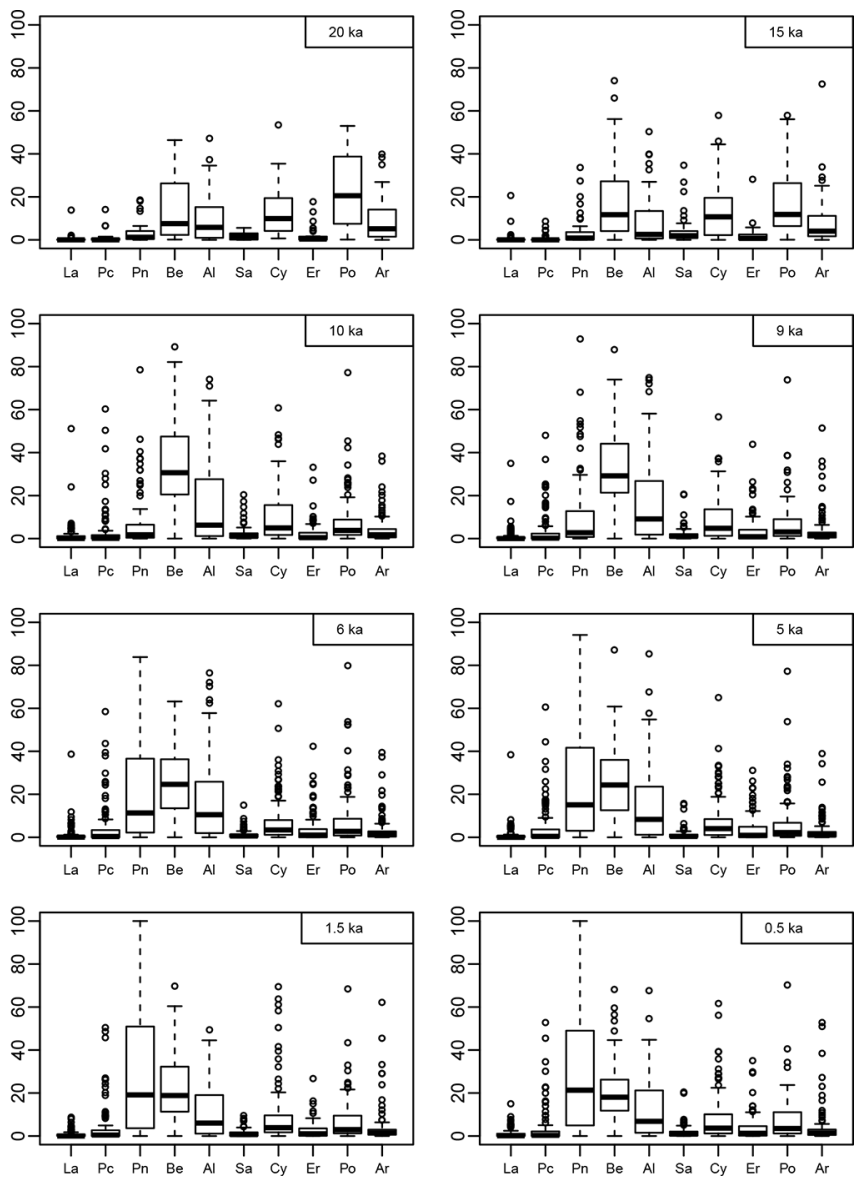

Figure 3. Boxplots of percentages of 10 major pollen taxa at all available sites at key time slices. La: Larix; Pc: Picea; Pn: Pinus; Be: Betula; Al: Alnus; Sa: Salix; Cy: Cyperaceae; Er: Ericaceae; Po: Poaceae; Ar: Artemisia.

and pollen count and pollen percentage for each sample are available at https://doi.org/10.1594/PANGAEA.898616 (Cao et al., 2019a).

\section{Summary}

We present a taxonomically harmonized and temporally standardized fossil pollen dataset of 173 palynological records with counts and percentages from Siberia and adjacent areas (northeastern Asia, $42-75^{\circ} \mathrm{N}, 50-180^{\circ} \mathrm{E}$ ).

Our open-access dataset is a key component that can help provide quantitative estimates of vegetation or climate, which can be used to validate palaeo-simulation results of general circulation models for the Northern Hemisphere. 


\section{Appendix A}

Table A1. Details of the fossil pollen records in the Siberian pollen dataset. NA - not available.

\begin{tabular}{|c|c|c|c|c|c|c|c|c|c|c|c|c|}
\hline ID & Site & $\begin{array}{l}\text { Lat. } \\
\left({ }^{\circ}\right)\end{array}$ & $\begin{array}{r}\text { Long. } \\
\left({ }^{\circ}\right)\end{array}$ & $\begin{array}{r}\text { Elev. } \\
(\mathrm{m})\end{array}$ & $\begin{array}{l}\text { Archive } \\
\text { type }\end{array}$ & $\begin{array}{l}\text { Data } \\
\text { type }\end{array}$ & Source & $\begin{array}{l}\text { Dating } \\
\text { method }\end{array}$ & $\begin{array}{l}\text { No. of dates \& } \\
\text { material code }\end{array}$ & $\begin{array}{r}\text { Time span } \\
\text { (ka BP) }\end{array}$ & $\begin{array}{r}\text { Res. } \\
(\mathrm{yr})\end{array}$ & Reference \\
\hline 1 & Pobochnoye & 53.03 & 51.84 & 58 & Peat sediment & digitized & - & ${ }^{14} \mathrm{C}$ & $10 \mathrm{C}+6 \mathrm{E}$ & $14.4-0$ & 540 & Kremenetski et al. (1999) \\
\hline 2 & Novienky peat & 52.24 & 54.75 & 197 & Peat sediment & counts & EPD, Pan & ${ }^{14} \mathrm{C}$ & $1 \mathrm{U}$ & $4.5-0$ & 270 & López-García et al. (2003) \\
\hline 3 & Ust'Mashevskoe & 56.32 & 57.88 & 220 & Peat sediment & counts & EPD, Pan & ${ }^{14} \mathrm{C}$ & $5 \mathrm{C}$ & $7.8-00$ & 150 & Panova et al. (1996) \\
\hline 4 & Aral Sea & 44.42 & 59.98 & 53 & Lake sediment & counts & EPD, Pan & ${ }^{14} \mathrm{C}$ & $4 \mathrm{U}$ & $8.7-0$ & 260 & Aleshinskaya (unpublished data) \\
\hline 5 & Fernsehsee Lake & 52.83 & 60.50 & 290 & Lake sediment & counts & From author & ${ }^{14} \mathrm{C}$ & $10 \mathrm{~A}$ & $9.1-0.4$ & 220 & Stobbe et al. (2015) \\
\hline 6 & Karasieozerskoe & 56.77 & 60.75 & 230 & Peat sediment & counts & EPD, Pan & ${ }^{14} \mathrm{C}$ & $3 \mathrm{~A}$ & $5.9-0.1$ & 190 & Panova (1997) \\
\hline 7 & Zaboinoe Lake & 55.53 & 62.37 & 275 & Lake sediment & counts & GPD, EPD, Pan & ${ }^{14} \mathrm{C}$ & $1 \mathrm{U}$ & $12.3-0.1$ & 220 & Khomutova and Pushenko (1995) \\
\hline 8 & Cape Shpindler & 69.72 & 62.80 & 20 & Fluvial sediment & counts & Pan & ${ }^{14} \mathrm{C}$ & $12 \mathrm{~A}$ & $15.8-0$ & 420 & Andreev et al. (2001) \\
\hline 9 & Mokhovoye & 53.77 & 64.25 & 178 & Peat sediment & counts & GPD, EPD, Pan & ${ }^{14} \mathrm{C}$ & $4 \mathrm{C}+1 \mathrm{E}$ & $6.0-0$ & 180 & Kremenetskii et al. (1994) \\
\hline 10 & Chernaya Gorka & 67.08 & 65.35 & 170 & Palsa sediment & digitized & - & ${ }^{14} \mathrm{C}$ & $1 \mathrm{~A}+3 \mathrm{C}$ & $10.1-6.9$ & 70 & Jankovská et al. (2006) \\
\hline 11 & Lake Lyadhej-To & 68.25 & 65.75 & 150 & Lake sediment & counts & Pan & ${ }^{14} \mathrm{C}$ & $14 \mathrm{~A}+6 \mathrm{E}$ & $12.5-0.3$ & 170 & Andreev et al. (2005) \\
\hline 12 & Chesnok peat & 60.00 & 66.50 & 42 & Peat sediment & counts & GPD, EPD, Pan & ${ }^{14} \mathrm{C}$ & $7 \mathrm{C}$ & $10.6-0.5$ & 280 & Volkova (1966) \\
\hline 13 & Baidara Gulf & 68.85 & 66.90 & 30 & Coastal sediment & counts & EPD, Pan & ${ }^{14} \mathrm{C}$ & $10 \mathrm{C}$ & $15.8-4.6$ & 170 & Andreev et al. (1998) \\
\hline 14 & Komaritsa peat & 57.50 & 69.00 & 42 & Peat sediment & counts & GPD, EPD, Pan & ${ }^{14} \mathrm{C}$ & $10 \mathrm{C}$ & $10.5-0.5$ & 350 & Volkova (1966) \\
\hline 15 & Demyanskoye & 59.50 & 69.50 & 65 & Fluvial sediment & counts & GPD, EPD, Pan & ${ }^{14} \mathrm{C}$ & $1 \mathrm{~A}$ & $50.3-22.3$ & 2000 & Bakhareva (1983) \\
\hline 16 & Nulsaveito & 67.53 & 70.17 & 57 & Peat sediment & counts & EPD, Pan & ${ }^{14} \mathrm{C}$ & $4 \mathrm{~A}+1 \mathrm{C}$ & $8.4-6.4$ & 70 & Panova (1990) \\
\hline 17 & Salym-Yugan & 60.02 & 72.08 & 56 & Peat sediment & digitized & - & ${ }^{14} \mathrm{C}$ & $5 \mathrm{C}$ & $10.1-0.2$ & 200 & Pitkänen et al. (2002) \\
\hline 18 & Nizhnevartovsk & 62.00 & 76.67 & 54 & Peat sediment & counts & GPD, EPD, Pan & ${ }^{14} \mathrm{C}$ & $3 \mathrm{~A}+7 \mathrm{C}$ & $11.1-0$ & 300 & Neustadt and Zelikson (1985) \\
\hline 19 & Nizhnevartovskoye & 61.25 & 77.00 & 55 & Peat sediment & counts & GPD, EPD, Pan & ${ }^{14} \mathrm{C}$ & $1 \mathrm{~A}+13 \mathrm{C}+1 \mathrm{E}$ & $12.6-0$ & 380 & Neishtadt (1976a) \\
\hline 20 & Entarnoye peat & 59.00 & 78.33 & 65 & Peat sediment & counts & GPD, EPD, Pan & ${ }^{14} \mathrm{C}$ & $5 \mathrm{C}$ & $14.9-0.9$ & 460 & Neishtadt (1976b) \\
\hline 21 & Lukaschin Yar & 61.00 & 78.50 & 65 & Peat sediment & counts & GPD, EPD, Pan & ${ }^{14} \mathrm{C}$ & $13 \mathrm{C}$ & $10.9-0.3$ & 430 & Neishtadt (1976a) \\
\hline 22 & Big Yarovoe Lake & 52.85 & 78.63 & 79 & Lake sediment & counts & From author & Biwa* & - & 4.3-0 & 190 & Rudaya et al. (2012) \\
\hline 23 & Sverdrup & 74.50 & 79.50 & 7 & Peat sediment & counts & GPD, EPD, Pan & ${ }^{14} \mathrm{C}$ & $3 \mathrm{C}$ & $13.4-11.1$ & 290 & Tarasov et al. (1995) \\
\hline 24 & BP99-04/06 & 73.41 & 79.67 & -32 & Marine sediment & counts & Pan & ${ }^{14} \mathrm{C}$ & $12 \mathrm{U}$ & $10.0-0.3$ & 190 & Kraus et al. (2003) \\
\hline 25 & Pur-Taz peatland & 66.70 & 79.73 & 50 & Peat sediment & counts & GPD, EPD, Pan & ${ }^{14} \mathrm{C}$ & $5 \mathrm{~A}$ & $10.3-4.7$ & 80 & Peteet et al. (1998) \\
\hline 26 & Petropavlovka & 58.33 & 82.50 & 100 & Peat sediment & counts & EPD, Pan & ${ }^{14} \mathrm{C}$ & $4 \mathrm{C}+1 \mathrm{E}$ & $10.5-0.1$ & 160 & Blyakharchuk (1989) \\
\hline 27 & Kalistratikha & 53.33 & 83.25 & 190 & Peat sediment & counts & GPD, EPD, Pan & ${ }^{14} \mathrm{C}$ & $4 \mathrm{~A}$ & $39.0-12.7$ & 1870 & Zudin and Votakh (1977) \\
\hline 28 & Tom' River peat & 56.17 & 84.00 & 100 & Peat sediment & counts & GPD & ${ }^{14} \mathrm{C}$ & $6 \mathrm{C}$ & $10.1-0.2$ & 390 & Arkhipov and Votakh (1980) \\
\hline 29 & Novouuspenka & 56.62 & 84.17 & 150 & Fluvial sediment & counts & EPD, Pan & ${ }^{14} \mathrm{C}$ & $5 \mathrm{C}$ & $5.3-0$ & 130 & Blyakharchuk (1989) \\
\hline 30 & Kirek Lake & 56.10 & 84.22 & 90 & Lake sediment & digitized & - & ${ }^{14} \mathrm{C}$ & $3 \mathrm{G}$ & $10.5-1.5$ & 190 & Blyakharchuk (2003) \\
\hline 31 & Zhukovskoye Mire & 56.33 & 84.83 & 106 & Peat sediment & counts & From author & ${ }^{14} \mathrm{C}$ & $9 \mathrm{C}+6 \mathrm{H}$ & $11.2-0$ & 130 & Borisova et al. (2011) \\
\hline 32 & Chaginskoe & 56.45 & 84.88 & 80 & Peat sediment & digitized & - & ${ }^{14} \mathrm{C}$ & $2 \mathrm{C}$ & $8.8-0$ & 320 & Blyakharchuk (2003) \\
\hline 33 & Karginskii Cape & 70.00 & 85.00 & 60 & Peat sediment & counts & GPD, EPD, Pan & ${ }^{14} \mathrm{C}$ & $13 \mathrm{C}$ & $8.9-3.5$ & 290 & Firsov et al. (1972) \\
\hline 34 & Ovrazhnoe & 56.25 & 85.17 & 110 & Peat sediment & counts & EPD, Pan & ${ }^{14} \mathrm{C}$ & $1 \mathrm{C}$ & $5.8-0.1$ & 230 & Blyakharchuk (1989) \\
\hline 35 & Bugristoye Bog & 58.25 & 85.17 & 100 & Peat sediment & counts & EPD, Pan & ${ }^{14} \mathrm{C}$ & $4 \mathrm{C}+1 \mathrm{E}$ & $11.5-5.0$ & 100 & Blyakharchuk (1989) \\
\hline 36 & Igarka peat & 67.48 & 86.50 & 2 & Peat sediment & counts & GPD, EPD, Pan & ${ }^{14} \mathrm{C}$ & $1 \mathrm{~A}+2 \mathrm{C}$ & $10.9-5.9$ & 230 & Kats (1953) \\
\hline 37 & Yenisei & 68.17 & 87.15 & 68 & Peat sediment & digitized & - & ${ }^{14} \mathrm{C}$ & $7 \mathrm{C}$ & $6.5-1.6$ & 110 & Andreev and Klimanov (2000) \\
\hline 38 & Teguldet & 57.33 & 88.17 & 150 & Peat sediment & counts & Pan & ${ }^{14} \mathrm{C}$ & $3 \mathrm{C}$ & $7.3-2.4$ & 90 & Blyakharchuk (1989) \\
\hline 39 & Maksimkin Yar & 58.33 & 88.17 & 150 & Peat sediment & counts & EPD, Pan & ${ }^{14} \mathrm{C}$ & $4 \mathrm{C}$ & $8.3-0.2$ & 170 & Blyakharchuk (1989) \\
\hline 40 & Lama Lake & 69.53 & 90.20 & 77 & Lake sediment & counts & From author & ${ }^{14} \mathrm{C}$ & $26 \mathrm{~A}+4 \mathrm{D}+4 \mathrm{E}$ & $19.5-0$ & 170 & Andreev et al. (2004) \\
\hline 41 & Levinson-Lessing Lake & 74.47 & 98.64 & NA & Lake sediment & counts & Pan & ${ }^{14} \mathrm{C}$ & $30 \mathrm{~A}+19 \mathrm{E}$ & $35.3-0$ & 390 & Andreev et al. (2003) \\
\hline 42 & LAO13-94 & 72.19 & 99.58 & 65 & Peat sediment & counts & Pan & ${ }^{14} \mathrm{C}$ & $2 \mathrm{C}+1 \mathrm{U}$ & $16.1-0$ & 1240 & Andreev et al. (2002) \\
\hline 43 & LAB2-95 & 72.38 & 99.86 & 65 & Peat sediment & counts & Pan & ${ }^{14} \mathrm{C}$ & $1 \mathrm{~A}+1 \mathrm{C}$ & $17.4-5.6$ & 980 & Andreev et al. (2002) \\
\hline 44 & Taymyr Lake_SAO4 & 74.53 & 100.53 & 47 & Lake sediment & counts & Pan & ${ }^{14} \mathrm{C}$ & $1 \mathrm{C}$ & $8.7-0.4$ & 600 & Andreev et al. (2003) \\
\hline 45 & Taymyr Lake_SAO1 & 74.55 & 100.53 & 47 & Lake sediment & counts & Pan & ${ }^{14} \mathrm{C}$ & $6 \mathrm{~A}+5 \mathrm{C}$ & $57.9-0$ & 1320 & Andreev et al. (2003) \\
\hline 46 & 11-CH-12A Lake & 72.40 & 102.29 & 60 & Lake sediment & counts & Pan & ${ }^{14} \mathrm{C}$ & $8 \mathrm{~A}+7 \mathrm{E}$ & $7.0-0.1$ & 110 & Klemm et al. (2016) \\
\hline 47 & Baikal-CON01-605-5 & 51.58 & 104.85 & 480 & Lake sediment & digitized & - & ${ }^{14} \mathrm{C}$ & $12 \mathrm{D}$ & $11.5-0$ & 130 & Demske et al. (2005) \\
\hline 48 & Baikal-CON01-605-3 & 51.59 & 104.85 & 480 & Lake sediment & digitized & - & ${ }^{14} \mathrm{C}$ & $5 \mathrm{D}$ & $17.7-0$ & 200 & Demske et al. (2005) \\
\hline 49 & Chernoe Lake & 50.95 & 106.63 & 500 & Lake sediment & counts & EPD, Pan & ${ }^{14} \mathrm{C}$ & $4 \mathrm{E}$ & $7-0.7$ & 620 & Vipper (2010) \\
\hline 50 & Khanda-1 & 55.44 & 107.00 & 840 & Peat sediment & counts & From author & ${ }^{14} \mathrm{C}$ & $3 \mathrm{C}$ & $3.1-0.3$ & 50 & Bezrukova et al. (2011) \\
\hline 51 & Khanda & 55.44 & 107.00 & 840 & Peat sediment & counts & From author & ${ }^{14} \mathrm{C}$ & $6 \mathrm{C}$ & $5.8-0$ & 140 & Bezrukova et al. (2011) \\
\hline 52 & Cheremushka Bog & 52.75 & 108.08 & 1500 & Peat sediment & digitized & - & ${ }^{14} \mathrm{C}$ & $6 \mathrm{C}$ & $33.5-0$ & 460 & Shichi et al. (2009) \\
\hline 53 & Okunaika & 55.52 & 108.47 & 802 & Peat sediment & counts & From author & ${ }^{14} \mathrm{C}$ & $6 \mathrm{C}$ & $8.3-2.0$ & 120 & Bezrukova et al. (2011) \\
\hline 54 & Baikal-CON01-603-5 & 53.95 & 108.91 & 480 & Lake sediment & digitized & - & ${ }^{14} \mathrm{C}$ & $10 \mathrm{D}$ & $15.8-0$ & 270 & Demske et al. (2005) \\
\hline 55 & Ukta Creek mouth & 55.80 & 109.70 & 906 & Peat sediment & counts & From author & ${ }^{14} \mathrm{C}$ & $3 U$ & $5.1-0$ & 160 & Bezrukova et al. (2006) \\
\hline 56 & Bolshoe Eravnoe Lake & 52.58 & 111.67 & 947 & Lake sediment & counts & EPD, Pan & ${ }^{14} \mathrm{C}$ & $3 \mathrm{E}$ & $7.3-0.2$ & 710 & Vipper (2010) \\
\hline 57 & Madjagara Lake & 64.83 & 120.97 & 160 & Lake sediment & counts & GPD, EPD, Pan & ${ }^{14} \mathrm{C}$ & $7 \mathrm{E}$ & $8.2-0.2$ & 120 & Andreev and Klimanov (1989) \\
\hline 58 & Khomustakh Lake & 63.82 & 121.62 & 120 & Lake sediment & counts & GPD, EPD, Pan & ${ }^{14} \mathrm{C}$ & $9 \mathrm{E}$ & $12.3-0.1$ & 170 & Andreev et al. (1989) \\
\hline 59 & Boguda Lake & 63.67 & 123.25 & 120 & Lake sediment & counts & GPD, EPD, Pan & ${ }^{14} \mathrm{C}$ & $7 \mathrm{E}$ & $10.9-0.4$ & 180 & Andreev et al. (1989) \\
\hline 60 & Barbarina Tumsa & 73.57 & 123.35 & 10 & Peat sediment & counts & Pan & ${ }^{14} \mathrm{C}$ & $4 \mathrm{C}$ & $4.9-0.3$ & 240 & Andreev et al. (2004) \\
\hline 61 & Lake Kyutyunda & 69.63 & 123.65 & 66 & Lake sediment & counts & Pan & ${ }^{14} \mathrm{C}$ & $10 \mathrm{E}$ & $10.8-0.3$ & 360 & Biskaborn et al. (2016) \\
\hline 62 & Suollakh & 57.05 & 123.85 & 816 & Peat sediment & counts & GPD, EPD, Pan & ${ }^{14} \mathrm{C}$ & $8 \mathrm{C}$ & $12.8-3.7$ & 180 & Andreev and Klimanov (1991) \\
\hline 63 & Derput Bog & 57.03 & 124.12 & 700 & Peat sediment & counts & GPD, EPD, Pan & ${ }^{14} \mathrm{C}$ & $1 \mathrm{~A}+4 \mathrm{C}$ & $11.7-0.8$ & 210 & Andreev and Klimanov (1991) \\
\hline 64 & Nikolay Lake & 73.67 & 124.25 & 35 & Lake sediment & counts & EPD, Pan & ${ }^{14} \mathrm{C}$ & $6 \mathrm{~A}$ & $12.5-0$ & 600 & Andreev et al. (2004) \\
\hline 65 & Dyanushka River & 65.04 & 125.04 & 123 & Fluvial sediment & counts & Pan & ${ }^{14} \mathrm{C}$ & $13 \mathrm{~A}$ & $12.6-0$ & 170 & Werner et al. (2010) \\
\hline 66 & Billyakh Lake & 65.27 & 126.75 & 340 & Lake sediment & counts & Pan & ${ }^{14} \mathrm{C}$ & $1 \mathrm{~A}+10 \mathrm{E}$ & $50.6-0.2$ & 470 & Müller et al. (2010) \\
\hline 67 & Billyakh Lake & 65.30 & 126.78 & 340 & Lake sediment & counts & Pan & ${ }^{14} \mathrm{C}$ & $7 \mathrm{~A}$ & $14.1-0$ & 180 & Müller et al. (2009) \\
\hline 68 & Dolgoe Ozero & 71.87 & 127.07 & 40 & Lake sediment & counts & From author & ${ }^{14} \mathrm{C}$ & $1 \mathrm{~A}+9 \mathrm{~B}$ & $15.3-0$ & 210 & Pisaric et al. (2001) \\
\hline 69 & Chabada Lake & 61.98 & 129.37 & 290 & Lake sediment & counts & GPD, EPD, Pan & ${ }^{14} \mathrm{C}$ & $15 \mathrm{U}$ & $13-0$ & 110 & Andreev and Klimanov (1989) \\
\hline
\end{tabular}


Table A1. Continued.

\begin{tabular}{|c|c|c|c|c|c|c|c|c|c|c|c|c|}
\hline ID & Site & $\begin{array}{r}\text { Lat. } \\
\left({ }^{\circ}\right)\end{array}$ & $\begin{array}{l}\text { Long. } \\
\left({ }^{\circ}\right)\end{array}$ & $\begin{array}{r}\text { Elev. } \\
(\mathrm{m})\end{array}$ & $\begin{array}{l}\text { Archive } \\
\text { type }\end{array}$ & $\begin{array}{l}\text { Data } \\
\text { type }\end{array}$ & Source & $\begin{array}{l}\text { Dating } \\
\text { method }\end{array}$ & $\begin{array}{l}\text { No. of dates \& } \\
\text { material code }\end{array}$ & $\begin{array}{r}\text { Time span } \\
(\mathrm{ka} \mathrm{BP})\end{array}$ & $\begin{array}{r}\text { Res. } \\
(\mathrm{yr})\end{array}$ & Reference \\
\hline 70 & Mamontovy Khayata & 71.77 & 129.45 & 0 & Coastal sediment & counts & Pan & ${ }^{14} \mathrm{C}$ & $40 \mathrm{~A}+24 \mathrm{C}$ & $58.4-0$ & 970 & Andreev et al. (2002) \\
\hline 71 & Nuochaga Lake & 61.30 & 129.55 & 260 & Lake sediment & counts & GPD, EPD, Pan & ${ }^{14} \mathrm{C}$ & $4 \mathrm{E}$ & $6.5-0$ & 140 & Andreev and Klimanov (1989) \\
\hline 72 & Tumannaya River & 42.32 & 130.73 & 4 & Fluvial sediment & counts & GPD & ${ }^{14} \mathrm{C}$ & $1 \mathrm{~F}$ & $14.4-0.1$ & 380 & Anderson et al. (2002) \\
\hline 73 & Amba River & 43.32 & 131.82 & 5 & Peat sediment & counts & GPD & ${ }^{14} \mathrm{C}$ & $1 \mathrm{~A}+1 \mathrm{C}$ & $4.2-2.0$ & 260 & Korotky et al. (1980) \\
\hline 74 & Paramonovskii Stream & 43.20 & 133.75 & 120 & Fluvial sediment & counts & GPD & ${ }^{14} \mathrm{C}$ & $2 \mathrm{~A}+1 \mathrm{E}$ & $32.2-0.6$ & 4530 & Korotky et al. (1993) \\
\hline 75 & Ovrazhnyi Stream-2 & 43.25 & 134.57 & 10 & Peat sediment & counts & GPD & ${ }^{14} \mathrm{C}$ & $3 \mathrm{~A}+1 \mathrm{C}$ & $36.0-0.4$ & 2250 & Korotky and Karaulova (1975) \\
\hline 76 & Selitkan-2 & 53.22 & 135.03 & 1300 & Peat sediment & counts & GPD & ${ }^{14} \mathrm{C}$ & $4 \mathrm{C}$ & $\begin{array}{r}3.4-1.9 \\
\end{array}$ & 260 & Volkov and Arkhipov (1978) \\
\hline 77 & Selitkan-1 & 53.22 & 135.05 & 1320 & Peat sediment & counts & GPD & ${ }^{14} \mathrm{C}$ & $6 \mathrm{C}$ & $7.9-0$ & 140 & Korotky et al. (1985) \\
\hline 78 & Selitkan-3 & 53.22 & 135.07 & 1310 & Peat sediment & counts & GPD & ${ }^{14} \mathrm{C}$ & $2 \mathrm{E}$ & $10.2-2.3$ & 790 & Korotky and Kovalyukh (1987) \\
\hline 79 & Bugutakh & 67.83 & 135.12 & 128 & Fluvial sediment & counts & GPD, EPD, Pan & ${ }^{14} \mathrm{C}$ & $1 \mathrm{~A}$ & $20.4-0$ & 1860 & Anderson et al. (2002) \\
\hline 80 & Betenkyos & 67.67 & 135.58 & 135 & Fluvial sediment & counts & GPD, EPD, Pan & ${ }^{14} \mathrm{C}$ & $1 \mathrm{~A}+1 \mathrm{E}$ & $2.2-0$ & 230 & Anderson et al. (2002) \\
\hline 81 & Adycha River & 67.75 & 135.58 & 130 & Fluvial sediment & counts & GPD & ${ }^{14} \mathrm{C}$ & $5 \mathrm{~A}$ & $9.2-3.7$ & 420 & Anderson et al. (2002) \\
\hline 82 & Ulakhan & 67.83 & 135.58 & 130 & Fluvial sediment & counts & GPD & ${ }^{14} \mathrm{C}$ & $3 \mathrm{C}$ & $8.6-5.7$ & 330 & Anderson et al. (2002) \\
\hline 83 & Kiya & 47.83 & 135.67 & 100 & Peat sediment & digitized & - & ${ }^{14} \mathrm{C}$ & $4 \mathrm{C}$ & $10.0-0.9$ & 210 & Bazarova et al. (2008) \\
\hline 84 & Laptev PM9462 & 74.30 & 136.00 & 0 & Marine sediment & digitized & - & ${ }^{14} \mathrm{C}$ & $12 \mathrm{U}$ & $9.3-0.2$ & 100 & Naidina and Bauch (2001) \\
\hline 85 & Khocho & 71.05 & 136.23 & 6 & Peat sediment & counts & GPD, EPD, Pan & ${ }^{14} \mathrm{C}$ & $1 \mathrm{C}$ & $10.4-0.4$ & 300 & Velichko et al. (1994) \\
\hline 86 & Samandon & 70.77 & 136.25 & 10 & Peat sediment & counts & GPD, EPD, Pan & ${ }^{14} \mathrm{C}$ & $3 \mathrm{~A}+8 \mathrm{C}+4 \mathrm{E}$ & $7.9-0.2$ & 280 & Velichko et al. (1994) \\
\hline 87 & Gur & 50.00 & 137.05 & 35 & Peat sediment & digitized & - & ${ }^{14} \mathrm{C}$ & $13 \mathrm{C}$ & $22.1-0$ & 340 & Mokhova et al. (2009) \\
\hline 88 & Gurskii peat & 50.07 & 137.08 & 15 & Peat sediment & counts & GPD & ${ }^{14} \mathrm{C}$ & $7 \mathrm{C}$ & $13.1-1.5$ & 380 & Korotky (1982) \\
\hline 89 & Siluyanov Yar & 46.13 & 137.83 & 20 & Fluvial sediment & counts & GPD & ${ }^{14} \mathrm{C}$ & $6 \mathrm{~A}$ & $12.8-4.9$ & 1130 & Korotky et al. (1988) \\
\hline 90 & Oumi & 48.22 & 138.40 & 990 & Peat sediment & counts & GPD & ${ }^{14} \mathrm{C}$ & $5 \mathrm{C}$ & $2.6-0.4$ & 80 & Anderson et al. (2002) \\
\hline 91 & Opasnaya River & 48.23 & 138.48 & 1320 & Peat sediment & counts & GPD & $14 \mathrm{C}$ & $7 \mathrm{C}$ & $13.3-6.7$ & 360 & Korotky et al. (1988) \\
\hline 92 & Venyukovka-2 & 47.03 & 138.58 & 6 & Peat sediment & counts & GPD & ${ }^{14} \mathrm{C}$ & $1 \mathrm{~A}+1 \mathrm{C}$ & $3.6-0.4$ & 140 & Korotky et al. (1980) \\
\hline 93 & Venyukovka-3 & 47.12 & 138.58 & 5 & Peat sediment & counts & GPD & ${ }^{14} \mathrm{C}$ & $1 \mathrm{~A}+2 \mathrm{C}$ & $5.8-3.2$ & 140 & Korotky et al. (1980) \\
\hline 94 & Kyurbe-Yuryakh-2 & 68.60 & 138.62 & 650 & Peat sediment & counts & GPD & ${ }^{14} \mathrm{C}$ & $4 \mathrm{C}$ & $8.8-2.6$ & 1530 & Anderson et al. (2002) \\
\hline 95 & Byllatskoye & 69.17 & 140.06 & 316 & Fluvial sediment & digitized & - & ${ }^{14} \mathrm{C}$ & $2 \mathrm{~A}$ & $28.6-2.8$ & 4300 & Anderson et al. (2002) \\
\hline 96 & Smorodinovoye Lake & 64.77 & 141.12 & 800 & Lake sediment & counts & GPD, EPD, Pan & ${ }^{14} \mathrm{C}$ & $6 \mathrm{~A}+5 \mathrm{~F}$ & $27.1-0$ & 360 & Anderson et al. (1998b) \\
\hline 97 & Izylmet'evskaya & 48.82 & 141.97 & 4 & Fluvial sediment & counts & GPD & ${ }^{14} \mathrm{C}$ & $2 \mathrm{~A}+2 \mathrm{E}+1 \mathrm{~F}$ & $4.3-2.8$ & 100 & Korotky et al. (1997a) \\
\hline 98 & Orokess River & 48.85 & 142.00 & 6 & Coastal sediment & counts & GPD & ${ }^{14} \mathrm{C}$ & $4 \mathrm{~A}+2 \mathrm{C}+3 \mathrm{~F}$ & $9.2-0.8$ & 320 & Korotky et al. (1997a) \\
\hline 99 & Nizmennyii Cape & 49.17 & 142.02 & 5 & Coastal sediment & counts & GPD & ${ }^{14} \mathrm{C}$ & $2 \mathrm{~A}$ & $5.9-0.3$ & 630 & Korotky et al. (1997a) \\
\hline 100 & Sergeevka River & 49.23 & 142.08 & 2 & Fluvial sediment & counts & GPD & ${ }^{14} \mathrm{C}$ & $2 \mathrm{C}+1 \mathrm{~F}$ & $2.3-0$ & 230 & Korotky et al. (1997b) \\
\hline 101 & Sergeevskii & 49.23 & 142.08 & 6 & Peat sediment & counts & GPD & ${ }^{14} \mathrm{C}$ & $8 \mathrm{~A}+1 \mathrm{C}$ & $8.4-2.2$ & 110 & Korotky et al. (1997b) \\
\hline 102 & Khoe, Sakhalin Island & 51.34 & 142.14 & 15 & Palaeosol & digitized & - & ${ }^{14} \mathrm{C}$ & $5 \mathrm{~A}+3 \mathrm{E}$ & $40.9-0$ & 360 & Leipe et al. (2015) \\
\hline 103 & Il'inka Terrace & 47.97 & 142.17 & 3 & Peat sediment & counts & GPD & ${ }^{14} \mathrm{C}$ & $2 \mathrm{C}+1 \mathrm{~F}$ & $2.6-1.1$ & 360 & Korotky et al. (1997a) \\
\hline 104 & Mereya River & 46.62 & 142.92 & 4 & Peat sediment & counts & GPD & ${ }^{14} \mathrm{C}$ & $2 \mathrm{C}+2 \mathrm{~F}$ & $42.0-0.8$ & 1530 & Anderson et al. (2002) \\
\hline 105 & Kuobakh-Baga River & 64.98 & 143.38 & 500 & Fluvial sediment & counts & GPD, EPD, Pan & ${ }^{14} \mathrm{C}$ & $5 \mathrm{~A}$ & $6.5-2.6$ & 350 & Anderson et al. (2002) \\
\hline 106 & Indigirka lowlands & 70.58 & 145.00 & 20 & Fluvial sediment & counts & GPD & ${ }^{14} \mathrm{C}$ & $3 \mathrm{~A}+1 \mathrm{~F}$ & $59.1-6.0$ & 1440 & Lozhkin (1998) \\
\hline 107 & Khlebnikova Stream & 43.75 & 145.62 & 3 & Peat sediment & counts & GPD & ${ }^{14} \mathrm{C}$ & $4 \mathrm{C}$ & $5.4-1.3$ & 290 & Korotky et al. (1995) \\
\hline 108 & Sernovodskii & 43.92 & 145.67 & 5 & Peat sediment & counts & GPD & ${ }^{14} \mathrm{C}$ & $1 \mathrm{C}$ & $3.5-0.7$ & 400 & Korotky et al. (1996) \\
\hline 109 & Lesnaya River & 44.00 & 145.75 & 6 & Peat sediment & counts & GPD & ${ }^{14} \mathrm{C}$ & $5 \mathrm{C}$ & 7.4-3.9 & 140 & Korotky et al. (1995) \\
\hline 110 & Seryebryanka Stream & 44.05 & 146.00 & 5 & Peat sediment & counts & GPD & ${ }^{14} \mathrm{C}$ & $4 \mathrm{C}+2 \mathrm{~F}$ & $5.9-0.1$ & 420 & Korotky et al. (1995) \\
\hline 111 & Kosmodem'yanskaya-2 & 44.10 & 146.05 & 6 & Peat sediment & counts & GPD & ${ }^{14} \mathrm{C}$ & $1 \mathrm{~A}+1 \mathrm{C}$ & $7.2-0.4$ & 570 & Korotky et al. (1995) \\
\hline 112 & Kosmodem'yanskaya-3 & 44.10 & 146.05 & 6 & Peat sediment & counts & GPD & ${ }^{14} \mathrm{C}$ & $1 \mathrm{~A}+2 \mathrm{C}$ & $7.0-5.6$ & 100 & Korotky et al. (1995) \\
\hline 113 & Kosmodem'yanskaya-1 & 44.10 & 146.07 & 6 & Peat sediment & counts & GPD & ${ }^{14} \mathrm{C}$ & $1 \mathrm{~A}+1 \mathrm{C}+1 \mathrm{E}$ & $6.6-2.4$ & 420 & Korotky et al. (1995) \\
\hline 114 & Berelyekh River & 63.28 & 147.75 & 800 & Peat sediment & counts & GPD, EPD, Pan & ${ }^{14} \mathrm{C}$ & $3 \mathrm{C}$ & $34.8-2.5$ & 1600 & Lozhkin et al. (1989) \\
\hline 115 & Vechernii River & 63.28 & 147.75 & 800 & Peat sediment & counts & GPD & ${ }^{14} \mathrm{C}$ & $2 \mathrm{~A}+5 \mathrm{C}$ & $6.1-0.3$ & 210 & Anderson et al. (2002) \\
\hline 116 & Gek Lake & 63.52 & 147.93 & 969 & Lake sediment & counts & GPD, EPD, Pan & ${ }^{14} \mathrm{C}$ & $8 \mathrm{~A}+1 \mathrm{~B}$ & $9.6-0$ & 440 & Stetsenko (1998) \\
\hline 117 & Kirgirlakh Stream_2 & 62.67 & 147.98 & 700 & Fluvial sediment & counts & GPD, EPD, Pan & ${ }^{14} \mathrm{C}$ & $4 \mathrm{~A}$ & $34.5-0.2$ & 2140 & Shilo et al. (1983) \\
\hline 118 & Kirgirlakh Stream_4 & 62.67 & 147.98 & 700 & Fluvial sediment & counts & GPD, EPD, Pan & ${ }^{14} \mathrm{C}$ & $4 \mathrm{~A}$ & $7.1-1.0$ & 610 & Shilo et al. (1983) \\
\hline 119 & Elgennya Lake & 62.08 & 149.00 & 1040 & Lake sediment & counts & GPD, EPD, Pan & ${ }^{14} \mathrm{C}$ & $6 \mathrm{~A}$ & $16.0-0$ & 310 & Lozhkin et al. (1996) \\
\hline 120 & Figurnoye Lake & 62.10 & 149.00 & 1053 & Lake sediment & counts & GPD & ${ }^{14} \mathrm{C}$ & $4 \mathrm{~A}$ & $1.3-0$ & 30 & Lozhkin et al. (1996) \\
\hline 121 & Jack London Lake & 62.17 & 149.50 & 820 & Lake sediment & counts & GPD & ${ }^{14} \mathrm{C}$ & $7 \mathrm{~F}$ & $19.5-0.2$ & 320 & Lozhkin et al. (1993) \\
\hline 122 & Rock Island Lake & 62.17 & 149.50 & 870 & Lake sediment & counts & GPD & ${ }^{14} \mathrm{C}$ & $2 \mathrm{E}$ & $6.6-0$ & 470 & Lozhkin et al. (1993) \\
\hline 123 & Sosednee Lake & 62.17 & 149.50 & 822 & Lake sediment & counts & GPD & ${ }^{14} \mathrm{C}$ & $4 \mathrm{E}+1 \mathrm{~F}$ & $26.3-0$ & 640 & Lozhkin et al. (1993) \\
\hline 124 & Oldcamp Lake & 62.04 & 149.59 & 853 & Lake sediment & counts & GPD, EPD, Pan & ${ }^{14} \mathrm{C}$ & $2 \mathrm{E}$ & $3.7-0$ & 370 & Anderson (unpublished data) \\
\hline 125 & Glukhoye Lake & 59.75 & 149.92 & 10 & Peat sediment & counts & GPD, EPD, Pan & ${ }^{14} \mathrm{C}$ & $5 \mathrm{C}$ & $9.4-3.4$ & 1000 & Lozhkin et al. (1990) \\
\hline 126 & Pepelnoye Lake & 59.85 & 150.62 & 115 & Lake sediment & counts & GPD, EPD, Pan & ${ }^{14} \mathrm{C}$ & $2 \mathrm{~A}$ & $4.3-0$ & 180 & Lozhkin et al. (2000b) \\
\hline 127 & Tanon River & 59.67 & 151.20 & 40 & Fluvial sediment & counts & GPD, EPD, Pan & ${ }^{14} \mathrm{C}$ & $6 \mathrm{~A}+4 \mathrm{C}+1 \mathrm{~F}$ & $42.4-6.6$ & 1240 & Lozhkin and Glushkova (1997a) \\
\hline 128 & Maltan River & 60.88 & 151.62 & 735 & Peat sediment & counts & GPD, EPD, Pan & ${ }^{14} \mathrm{C}$ & $4 \mathrm{~A}+7 \mathrm{C}$ & $12.0-9.4$ & 120 & Lozhkin and Glushkova (1997b) \\
\hline 129 & Chistoye Lake & 59.55 & 151.83 & 91 & Peat sediment & counts & EPD, Pan & ${ }^{14} \mathrm{C}$ & $5 \mathrm{C}$ & $7.0-0$ & 540 & Anderson et al. (1997) \\
\hline 130 & Lesnoye Lake & 59.58 & 151.87 & 95 & Lake sediment & counts & GPD & ${ }^{14} \mathrm{C}$ & $8 \mathrm{~A}$ & $15.5-0$ & 400 & Anderson et al. (1997) \\
\hline 131 & Elikchan 4 Lake & 60.75 & 151.88 & 810 & Lake sediment & counts & GPD, EPD, Pan & ${ }^{14} \mathrm{C}$ & $16 \mathrm{U}$ & $55.5-0$ & 440 & Lozhkin and Anderson (1995) \\
\hline 132 & Podkova Lake & 59.96 & 152.10 & 660 & Lake sediment & counts & GPD, EPD, Pan & ${ }^{14} \mathrm{C}$ & $5 \mathrm{~A}$ & $6.0-0$ & 220 & Anderson et al. (1997) \\
\hline 133 & Goluboye Lake & 61.12 & 152.27 & 810 & Lake sediment & counts & EPD, Pan & ${ }^{14} \mathrm{C}$ & $11 \mathrm{~A}+2 \mathrm{~B}$ & $9.7-0$ & 240 & Lozhkin et al. (2000a) \\
\hline 134 & Alut Lake & 60.14 & 152.31 & 480 & Lake sediment & counts & GPD & ${ }^{14} \mathrm{C}$ & $16 \mathrm{~A}+9 \mathrm{~B}$ & $50.4-0$ & 430 & Anderson et al. (1998a) \\
\hline 135 & Taloye Lake & 61.02 & 152.33 & 750 & Lake sediment & counts & GPD, EPD, Pan & ${ }^{14} \mathrm{C}$ & $7 \mathrm{~A}$ & $10.3-0$ & 290 & Lozhkin et al. (2000a) \\
\hline 136 & Julietta Lake & 61.34 & 154.56 & 880 & Lake sediment & counts & From author & ${ }^{14} \mathrm{C}$ & $2 \mathrm{~A}+4 \mathrm{E}+1 \mathrm{I}$ & $36.1-1.4$ & 270 & Anderson et al. (2010) \\
\hline 137 & Pernatoye Lake & 50.04 & 155.40 & 6 & Lake sediment & counts & From author & ${ }^{14} \mathrm{C}$ & $6 \mathrm{~A}+1 \mathrm{E}$ & $10.1-0.1$ & 160 & Anderson et al. (2015) \\
\hline 138 & East Siberian Sea 11 & 71.07 & 156.25 & 8 & Peat sediment & counts & GPD, Pan & ${ }^{14} \mathrm{C}$ & $2 \mathrm{~A}+2 \mathrm{C}$ & $9.5-4.5$ & 550 & Lozhkin et al. (1975) \\
\hline 139 & Kur peat & 69.97 & 156.37 & 47 & Peat sediment & counts & GPD, EPD, Pan & ${ }^{14} \mathrm{C}$ & $1 \mathrm{~A}+4 \mathrm{C}$ & $11.7-7.5$ & 430 & Lozhkin and Vazhenina (1987) \\
\hline 140 & East Siberian Sea coast & 71.07 & 156.50 & 9 & Peat sediment & counts & GPD & ${ }^{14} \mathrm{C}$ & $1 \mathrm{C}$ & $13.0-1.7$ & 1600 & Anderson et al. (2002) \\
\hline 141 & Kurop7 & 70.67 & 156.75 & 7 & Peat sediment & counts & GPD, EPD, Pan & ${ }^{14} \mathrm{C}$ & $3 \mathrm{C}$ & $5.7-0.4$ & 760 & Anderson et al. (2002) \\
\hline 142 & Sokoch Lake & 53.25 & 157.75 & 495 & Lake sediment & digitized & - & ${ }^{14} \mathrm{C}$ & $8 \mathrm{E}$ & $9.7-0.3$ & 250 & Dirksen et al. (2012) \\
\hline 143 & Stadukhinskaya-1 & 68.67 & 159.50 & 12 & Fluvial sediment & counts & GPD, EPD, Pan & ${ }^{14} \mathrm{C}$ & $4 \mathrm{C}$ & $9.5-7.2$ & 210 & Lozhkin and Prokhorova (1982) \\
\hline
\end{tabular}


Table A1. Continued.

\begin{tabular}{|c|c|c|c|c|c|c|c|c|c|c|c|c|}
\hline ID & Site & $\begin{array}{l}\text { Lat. } \\
\left({ }^{\circ}\right)\end{array}$ & $\begin{array}{l}\text { Long. } \\
\left({ }^{\circ}\right)\end{array}$ & $\begin{array}{r}\text { Elev. } \\
(\mathrm{m})\end{array}$ & $\begin{array}{l}\text { Archive } \\
\text { type }\end{array}$ & $\begin{array}{l}\text { Data } \\
\text { type }\end{array}$ & Source & $\begin{array}{l}\text { Dating } \\
\text { method }\end{array}$ & $\begin{array}{l}\text { No. of dates \& } \\
\text { material code }\end{array}$ & $\begin{array}{r}\text { Time span } \\
\text { (ka BP) }\end{array}$ & $\begin{array}{r}\text { Res. } \\
(\mathrm{yr})\end{array}$ & Reference \\
\hline 144 & Stadukhinskaya-2 & 68.67 & 159.50 & 5 & Fluvial sediment & counts & GPD, EPD, Pan & ${ }^{14} \mathrm{C}$ & $2 \mathrm{C}$ & $1.0-0$ & 180 & Lozhkin and Prokhorova (1982) \\
\hline 145 & Two-Yurts Lake-3 & 56.82 & 160.04 & 275 & Lake sediment & percent & Pan & ${ }^{14} \mathrm{C}$ & $5 \mathrm{~A}$ & $6.0-2.8$ & 140 & Hoff et al. (2015) \\
\hline 146 & Two-Yurts Lake-2 & 56.82 & 160.07 & 275 & Lake sediment & percent & Pan & ${ }^{14} \mathrm{C}$ & $5 \mathrm{~A}$ & $2.5-0.1$ & 130 & Hoff et al. (2015) \\
\hline 147 & Two-Yurts Lake-5 & 56.82 & 160.07 & 275 & Lake sediment & percent & Pan & ${ }^{14} \mathrm{C}$ & $5 \mathrm{~A}$ & $4.4-2.5$ & 120 & Hoff et al. (2015) \\
\hline 148 & Cherny Yar & 56.07 & 161.00 & 148 & Peat sediment & counts & GPD, EPD, Pan & ${ }^{14} \mathrm{C}$ & $1 \mathrm{C}+1 \mathrm{E}$ & $13.0-0.5$ & 830 & Osipova (unpublished data) \\
\hline 149 & Penzhinskaya Gulf & 62.42 & 165.42 & 32 & Peat sediment & counts & GPD, EPD, Pan & ${ }^{14} \mathrm{C}$ & $2 \mathrm{C}$ & $8.9-3.4$ & 500 & Ivanov et al. (1984) \\
\hline 150 & Enmynveem River1 & 68.17 & 165.93 & 400 & Peat sediment & counts & GPD, EPD, Pan & ${ }^{14} \mathrm{C}$ & $2 \mathrm{C}+2 \mathrm{~F}$ & $36.4-9.3$ & 2470 & Lozhkin et al. (1988) \\
\hline 151 & Enmynveem River2 & 68.25 & 166.00 & 500 & Peat sediment & counts & GPD, EPD, Pan & ${ }^{14} \mathrm{C}$ & $4 \mathrm{C}$ & $10.7-4.0$ & 420 & Anderson et al. (2002) \\
\hline 152 & Ledovyi Obryu & 64.10 & 171.18 & 44 & Lake sediment & counts & GPD, EPD, Pan & ${ }^{14} \mathrm{C}$ & $3 \mathrm{~A}+3 \mathrm{C}+1 \mathrm{~F}$ & $19.9-9.7$ & 1140 & Lozhkin et al. (2000c) \\
\hline 153 & Enmyvaam River & 67.42 & 172.08 & 490 & Peat sediment & counts & GPD, EPD, Pan & ${ }^{14} \mathrm{C}$ & $1 \mathrm{~A}+4 \mathrm{C}$ & $10.6-4.3$ & 630 & Lozhkin and Vazhenina (1987) \\
\hline 154 & El'gygytgyn Lake & 67.50 & 172.10 & (170) & Lake sediment & percent & Pan & polarity & - & $20.2-1.5$ & 650 & Melles et al. (2012) \\
\hline 155 & El'gygytgyn Lake P1 & 67.37 & 172.22 & 561 & Palaeosol & counts & From author & ${ }^{14} \mathrm{C}$ & $11 \mathrm{~A}$ & $12.9-3.1$ & 580 & Andreev et al. (2012) \\
\hline 156 & El'gygytgyn Lake P2 & 67.55 & 172.13 & 542 & Palaeosol & counts & From author & ${ }^{14} \mathrm{C}$ & $9 A+1 E$ & $16.6-0$ & 470 & Andreev et al. (2012) \\
\hline 157 & Melkoye Lake & 64.86 & 175.23 & 36 & Lake sediment & counts & From author & ${ }^{14} \mathrm{C}$ & $21 \mathrm{E}$ & $39.1-0$ & 1260 & Lozhkin and Anderson (2013) \\
\hline 158 & Sunset Lake & 64.84 & 175.30 & 36 & Lake sediment & counts & From author & ${ }^{14} \mathrm{C}$ & $7 \mathrm{~A}$ & $14.0-0$ & 260 & Lozhkin and Anderson (2013) \\
\hline 159 & Malyi Krechet Lake & 64.80 & 175.53 & 32 & Lake sediment & counts & From author & ${ }^{14} \mathrm{C}$ & $12 \mathrm{~A}$ & $9.6-0$ & 400 & Lozhkin and Anderson (2013) \\
\hline 160 & Patricia Lake & 63.33 & 176.50 & 121 & Lake sediment & counts & From author & ${ }^{14} \mathrm{C}$ & $3 A+7 E$ & $19.1-0$ & 290 & Anderson and Lozhkin (2015) \\
\hline 161 & Gytgykai Lake & 63.42 & 176.57 & 102 & Lake sediment & counts & GPD, EPD, Pan & ${ }^{14} \mathrm{C}$ & $1 \mathrm{~A}+8 \mathrm{E}$ & $32.3-0$ & 470 & Lozhkin et al. (1998) \\
\hline 162 & Amguema River 1 & 67.75 & 178.70 & 175 & Fluvial sediment & counts & GPD & ${ }^{14} \mathrm{C}$ & $2 \mathrm{C}$ & $23.8-1.6$ & 5550 & Lozhkin et al. (1995) \\
\hline 163 & Amguema River 2 & 67.67 & 178.60 & 87 & Fluvial sediment & counts & GPD & ${ }^{14} \mathrm{C}$ & $2 \mathrm{~A}$ & $3.2-0.1$ & 390 & Lozhkin et al. (1995) \\
\hline 164 & Blossom Cape & 70.68 & 178.95 & 6 & Peat sediment & counts & GPD, EPD, Pan & ${ }^{14} \mathrm{C}$ & $1 \mathrm{C}$ & $13.8-0.2$ & 3400 & Oganesyan et al. (1993) \\
\hline 165 & Wrangel Island_JLL & 70.83 & -179.8 & 7 & Lake sediment & counts & GPD & ${ }^{14} \mathrm{C}$ & $5 \mathrm{~A}+1 \mathrm{E}$ & $16.1-0.3$ & 790 & Lozhkin et al. (2001) \\
\hline 166 & Wrangel Island_wr12 & 71.17 & -179.8 & 200 & Peat sediment & counts & GPD & ${ }^{14} \mathrm{C}$ & $17 \mathrm{~A}+3 \mathrm{C}$ & $13.7-10.2$ & 110 & Lozhkin et al. (2001) \\
\hline 167 & Neizvestnaya & 71.55 & -179.4 & 3 & Peat sediment & counts & EPD, Pan & ${ }^{14} \mathrm{C}$ & $1 \mathrm{C}$ & $5.2-1.2$ & 1000 & Oganesyan et al. (1993) \\
\hline 168 & Kresta Gulf & 66.00 & -179.0 & 5 & Peat sediment & counts & GPD, EPD, Pan & ${ }^{14} \mathrm{C}$ & $1 \mathrm{~A}+1 \mathrm{C}$ & $9.3-3.4$ & 580 & Ivanov (1986) \\
\hline 169 & Konergino & 65.90 & -178.9 & 10 & Peat sediment & counts & GPD, EPD, Pan & ${ }^{14} \mathrm{C}$ & $1 \mathrm{C}$ & $9.8-0$ & 900 & Ivanov et al. (1984) \\
\hline 170 & Dlinnoye Lake & 67.75 & -178.8 & 280 & Lake sediment & counts & GPD & ${ }^{14} \mathrm{C}$ & $3 \mathrm{~A}$ & $1.3-0$ & 130 & Anderson et al. (2002) \\
\hline 171 & Dikikh Olyenyeii Lake & 67.75 & -178.8 & 300 & Lake sediment & counts & EPD, Pan & ${ }^{14} \mathrm{C}$ & $1 \mathrm{~A}+4 \mathrm{C}$ & $50.3-0$ & 1050 & Anderson et al. (2002) \\
\hline 172 & Arakamchechen Island & 64.75 & -172.1 & 7 & Peat sediment & counts & GPD, EPD, Pan & ${ }^{14} \mathrm{C}$ & $1 \mathrm{C}$ & $11.5-0$ & 1050 & Ivanov (1986) \\
\hline 173 & Lorino & 65.50 & -171.7 & 12 & Peat sediment & counts & GPD & ${ }^{14} \mathrm{C}$ & $3 \mathrm{C}$ & $17.9-5.1$ & 850 & Ivanov (1986) \\
\hline
\end{tabular}

* Indicates the inclination of age-depth model with Lake Biwa. Elev. indicates elevation. Res. (year) indicates the temporal resolution. GPD: Global Pollen Database; EPD:

European Pollen Database; Pan: PANGAEA. Material codes for radiocarbon dating: $\mathrm{A}=$ terrestrial plant macrofossil; $\mathrm{B}=$ non-terrestrial plant macrofossil; C = peat-gyttja bulk; $\mathrm{D}=$ pollen $\mathrm{E}=$ total organic matter from silt $\mathrm{F}=$ animal remains and shells; $\mathrm{G}=$ charcoal $\mathrm{H}=\mathrm{CaCO}_{3} ; \mathrm{U}=$ unknown. 


\section{Appendix B}

Table B1. Pollen taxa used in the dataset and their corresponding original Latin names.

\begin{tabular}{|c|c|}
\hline Standardized pollen name & Original pollen name \\
\hline Abies & Abies, Abies sibirica \\
\hline Acer & Acer \\
\hline Alnus (shrub) & $\begin{array}{l}\text { Alnaster, Alnaster fruticosa, Alnus cf. fruticosa, Alnus viridis, Alnus viridis ssp. fruticosa, Alnus viridis } \\
\text { type, Duschekia fruticosa }\end{array}$ \\
\hline Alnus (tree) & Alnus cf. hirsuta, Alnus glutinosa, Alnus hirsuta, Alnus incana \\
\hline Alnus (undiff.) & Alnus, Alnus undiff. \\
\hline Apiaceae & Apiaceae, Bupleurum, Heracleum, Umbelliferae, Umbelliferae undiff. \\
\hline Araliaceae & Aralia, Araliaceae \\
\hline Artemisia & Artemisia, Artemisia tilesii, Artemisia undiff. \\
\hline Asteraceae (non-Artemisia) & $\begin{array}{l}\text { Achillea, Anthemis, Aster, Asteraceae, Asteraceae cichorioideae, Asteraceae liguliflorae, Asteraceae } \\
\text { subfam. Asteroideae, Asteraceae subfam. cichorioideae, Asteraceae tubuliflorae, Centaurea cyanus, } \\
\text { Cirsium, Compositae, Compositae subfam. Asteroideae, Compositae subfam. Asteroideae undiff., } \\
\text { Compositae subfam. Cichorioideae, Lactuca type, Matricaria, Saussurea, Senecio, Serratula, Tarax- } \\
\text { acum }\end{array}$ \\
\hline Betula (shrub) & $\begin{array}{l}\text { Betula (shrub), Betula cf. B. fruticosa, Betula cf. B. nana, Betula cf.nana, Betula divaricata, Betula } \\
\text { fruticosa, Betula nana, Betula nana ssp. exilis, Betula nana ssp. nana, Betula ovalifolia, Betula sect. } \\
\text { Fruticosae, Betula sect. Nanae, Betula sect. Nanae/Fruticosae }\end{array}$ \\
\hline Betula (tree) & $\begin{array}{l}\text { Betula alba type, Betula cf. B. pendula, Betula cf. alba, Betula costata, Betula dahurica, Betula ermanii, } \\
\text { Betula pendula, Betula platyphylla, Betula pubescens, Betula schmidtii, Betula sect. Albae, Betula sect. } \\
\text { Betula, Betula sect. Costatae }\end{array}$ \\
\hline Betula (undiff.) & Betula, Betula undiff., Betulaceae undiff. \\
\hline Boraginaceae & Boraginaceae, Lithospermum type \\
\hline Brassicaceae & Brassicaceae, Brassicaceae undiff., Cardamine, Cruciferae, Crucififerae, Draba \\
\hline Campanulaceae & Campanulaceae \\
\hline Cannabis & Cannabaceae, Cannabis \\
\hline Caprifoliaceae & $\begin{array}{l}\text { Caprifoliaceae, Caprifoliaceae undiff., Diervilla, Knautia, Linnaea borealis, Lonicera, Sambucus, } \\
\text { Viburnum }\end{array}$ \\
\hline Carpinus & Carpinus, Carpinus cordata, Carpinus betulus \\
\hline Carya & Carya \\
\hline Caryophyllaceae & $\begin{array}{l}\text { Caryophyllaceae, Caryophyllaceae Sf. Silenoideae-type, Caryophyllaceae undiff., Cerastium, Gyp- } \\
\text { sophila repens type, Illecebrum verticillatum, Lychnis type, Minuartia, Silene, Stellaria holostea }\end{array}$ \\
\hline Castanea & Castanea \\
\hline Cedrus & Cedrus \\
\hline Celastraceae & Celastraceae, Euonymus \\
\hline Celtis & Celtis \\
\hline Cerealia+large Poaceae & Cerealia, Hordeum, Triticum type \\
\hline Chenopodiaceae & Chenopodiaceae, Chenopodiaceae/Amaranthaceae \\
\hline Convolvulaceae & Convolvulaceae \\
\hline Cornus & Cornus, Cornus suecica \\
\hline Corylus & Corylus \\
\hline Crassulaceae & Crassulaceae, Mentanthes trifoliata, Sedum \\
\hline Cupressaceae (other) & Cupressaceae \\
\hline Cyperaceae & Cyperaceae \\
\hline Dacrydium & Dacrydium \\
\hline Dipsacaceae & Dipsacaceae, Succisa \\
\hline Droseraceae & Drosera, Droseraceae \\
\hline Elaeagnus & Elaeagnus \\
\hline Ephedra & Ephedra, Ephedra distachya, Ephedra distachya +fragilis, Ephedra fragilis, Ephedra monosperma \\
\hline Ericaceae & $\begin{array}{l}\text { Calluna, Cassiope, Empetrum, Ericaceae, Ericaceae undiff., Ericales, Ericales undiff., Ledum, Rhodo- } \\
\text { dendron, Vaccinium }\end{array}$ \\
\hline Euphorbiaceae & Euphorbia, Euphorbiaceae \\
\hline
\end{tabular}


Table B1. Continued.

\begin{tabular}{|c|c|}
\hline Standardized pollen name & Original pollen name \\
\hline Fabaceae (herb) & Trifolium \\
\hline Fabaceae (shrub) & Astragalus \\
\hline Fabaceae (undiff.) & Fabaceae, Fabaceae undiff., Leguminosae, Papilionaceae \\
\hline Fagus & Fagus \\
\hline Gentianaceae & Gentiana, Gentianaceae, Gentianaceae undiff. \\
\hline Geraniaceae & Geraniaceae, Geranium \\
\hline Hippophäe & Hippophäe rhamnoides \\
\hline Humulus & Humulus \\
\hline Ilex & Ilex \\
\hline Impatiens & Impatiens noli-tangere \\
\hline Iridaceae & Iridaceae \\
\hline Juglans & Juglans \\
\hline Juncaceae & Juncaceae \\
\hline Juniperus & Juniperus \\
\hline Koenigia & Koenigia islandica \\
\hline Lamiaceae & Labiatae, Lamiaceae, Lamiaceae undiff., Mentha type \\
\hline Larix & Larix, Larix dahurica, Larix gmelinii, Larix sibirca \\
\hline Liliaceae & Allium, Liliaceae, Lloydia, Polygonatum, Tofieldia, Veratrum, Zigadenus \\
\hline Linaceae & Linaceae \\
\hline Lythraceae & Lythraceae, Lythrum \\
\hline Malvaceae & Malvaceae \\
\hline Myrica & Myrica \\
\hline Oenotheraceae & $\begin{array}{l}\text { Chamaenerium, Circaea, Circaea alpina, Epilobium, Epilobium angustifolium, Epilobium latifolium, } \\
\text { Epilobium undiff., Onagraceae, Onagraceae undiff. }\end{array}$ \\
\hline Oleaceae (temperate) & Fraxinus, Fraxinus mandschurica \\
\hline Oleaceae (undiff.) & Oleaceae, Oleaceae undiff., Syringa \\
\hline Orchidaceae & Orchidaceae \\
\hline Oxalidaceae & Oxalidaceae \\
\hline Papaveraceae & Corydalis, Papaver, Papaveraceae \\
\hline Phellodendron & Phellodendron \\
\hline Picea & $\begin{array}{l}\text { Picea, Picea abies ssp. obovata, Picea obovata, Picea sect. Eupicea, Picea sect. Omorica, Picea undiff., } \\
\text { Picea/Pinus undiff. }\end{array}$ \\
\hline Pinguicula & Pinguicula \\
\hline Pinus (Diploxylon) & Pinus (Diploxylon), Pinus subgen. Pinus, Pinus subg. Pinus undiff., Pinus sylvestris \\
\hline Pinus (Haploxylon) & $\begin{array}{l}\text { Pinus (Haploxylon), Pinus cembra, Pinus koraiensis, Pinus pumila, Pinus sibirica, Pinus sibirica type, } \\
\text { Pinus subgen. Strobus, Pinus subgen. Strobus undiff., Pinus subgen. Haploxylon, Pinus subsect. Cem- } \\
\text { brae undiff. }\end{array}$ \\
\hline Pinus (undiff.) & Pinaceae, Pinaceae undiff., Pinus, Pinus undiff. \\
\hline Plantago & Plantaginaceae, Plantago \\
\hline Plumbaginaceae & Armeria, Armeria maritima type, Goniolimon, Limonium, Plumbaginaceae \\
\hline Poaceae (wildgrass) & Gramineae, Poaceae, Stipa \\
\hline Podocarpus & Podocarpus \\
\hline Polemoniaceae & $\begin{array}{l}\text { Helianthemum, Phlox, Phlox sibirica, Polemoniaceae, Polemoniaceae undiff., Polemonium, Polemo- } \\
\text { nium acutiflorum, Polemonium boreale }\end{array}$ \\
\hline Polygala & Polygala \\
\hline Polygonaceae (other) & Oxyria, Oxyria digyna, Polygonaceae, Polygonaceae undiff. \\
\hline Polygonum & $\begin{array}{l}\text { Polygonum, Polygonum alaskanum, Polygonum amphibium, Polygonum aviculare, Polygonum bistorta, } \\
\text { Polygonum bistortoides type, Polygonum czukavinae, Polygonum ellipticum, Polygonum laxmanii, Poly- } \\
\text { gonum sect. Aconogonon, Polygonum sect. Bistorta, Polygonum sect. Persicaria, Polygonum triptero- } \\
\text { carpum, Polygonum undiff., Polygonum viviparum }\end{array}$ \\
\hline Populus & Populus \\
\hline Portulacaceae & $\begin{array}{l}\text { Claytonia, Claytonia acutifolia, Claytonia arctica, Claytonia sarmentosa, Claytonia sibirica, Claytonia } \\
\text { undiff., Claytoniella vassilievii, Portulacaceae, Portulacaceae undiff. }\end{array}$ \\
\hline Primulaceae & Androsace, Androsaceae, Lysimachia, Primula, Primulaceae, Primulaceae undiff. \\
\hline
\end{tabular}


Table B1. Continued.

\begin{tabular}{ll}
\hline Standardized pollen name & Original pollen name \\
\hline Pterocarya & Pterocarya \\
Pyrolaceae & Pyrolaceae \\
Quercus (deciduous) & Quercus dentata, Quercus mongolica \\
Quercus (undiff.) & Quercus, Quercus undiff. \\
Ranunculaceae (other) & Anemone, Anemone nemorosa, Caltha palustris, Delphinium, Hepatica, Pulsatilla, Ranunculaceae, Ra- \\
& nunculaceae undiff., Ranunculus, Trollius \\
Rhamnus & Rhamnus \\
Ribes & Ribes, Ribes rubrum type \\
Rosaceae & Comarum palustre, Dryas, Dryas octopetala, Filipendula, Filipendula ulmaria, Potentilla, Rosaceae, \\
& Rosaceae subf. Maloideae, Rosaceae undiff., Rubus, Rubus atcticus, Rubus chamaemorus, Sanguisorba, \\
& Sanguisorba officinalis, Sieversia type, Sorbus aucuparia, Spiraea \\
Rubiaceae & Galium, Rubiaceae \\
Rumex & Rumex, Rumex aquatilis, Rumex undiff., Rumex/Oxyria, Rumex/Oxyria digyna \\
Salix & Salix \\
Saxifragaceae (herb) & Parnassia, Parnassia palustris, Saxifraga, Saxifraga cernua, Saxifraga gramulata type, Saxifraga hi- \\
& eracifolia, Saxifraga nivalis type, Saxifraga oppositifolia, Saxifraga sp., Saxifraga stellaris type, Sax- \\
Saxifragaceae (undiff.) & ifraga tricuspidata, Saxifraga undiff. \\
Scrophulariaceae & Saxifragaceae, Saxifragaceae undiff. \\
Thalictrum & Castilleja, Lagotis, Pedicularis, Scrophulariaceae, Scrophulariaceae undiff. \\
Tilia & Thalictrum \\
Tsuga & Tilia \\
Ulmus & Tsuga, Tsuga canadensis, Tsuga diversifolia, Tsuga undiff. \\
Urtica & Ulmus, Ulmus glabra, Ulmus minor, Ulmus sp. \\
Urticaceae (non-Urtica) & Urtica \\
Valerianaceae & Urticaceae \\
& Patrinia, Valeriana, Valeriana capitata, Valeriana officinalis, Valeriana undiff., Valerianaceae, Valeri- \\
Violaceae & anaceae undiff. \\
\hline & Violaceae \\
\hline
\end{tabular}


Author contributions. $\mathrm{UH}$ and $\mathrm{XC}$ designed the pollen dataset. $\mathrm{XC}$ and FT compiled the standardization for the dataset and wrote the draft. Other authors provided pollen data and all authors discussed the results and contributed to the final paper.

Competing interests. The authors declare that they have no conflict of interest.

Special issue statement. This article is part of the special issue "Paleoclimate data synthesis and analysis of associated uncertainty (BG/CP/ESSD inter-journal SI)". It is not associated with a conference.

Acknowledgements. The authors would like to express their gratitude to all the palynologists who, either directly or indirectly, contributed their pollen records to the dataset or accessible databases.

Financial support. This data collection and research were supported by the German Research Foundation (DFG), Palmod project (German Ministry of Science and Education), the GlacialLegacy project (consolidator grant of the European Research Council of UH, grant agreement no. 772852), and the Russian Fund for Basic Research (for AVL, research project no. 19-05-00477).

Review statement. This paper was edited by Michal Kucera and reviewed by Patrick Bartlein and one anonymous referee.

\section{References}

Anderson, P., Minyuk, P., Lozhkin, A., Cherepanova, M., Borkhodoev, V., and Finney, B.: A multiproxy record of Holocene environmental changes from the northern Kuril Islands (Russian Far East), J. Paleolimnol., 54, 379-393, https:/dor.org/10.1007/s10933-015-9858-y, 2015.

Anderson, P. M. and Lozhkin, A. V.: LateQuaternary Vegetation and Climate of Siberia and the Russian Far East (Palynological and Radiocarbon Database), North East Science Center, Far East Branch, Russian Academy of Sciences, Magadan, Russia, 2002.

Anderson, P. M. and Lozhkin, A. V.: Late Quaternary vegetation of Chukotka (Northeast Russia), implications for Glacial and Holocene environments of Beringia, Quaternary Sci. Rev., 107, 112-128, https://doi.org/10.1016/j.quascirev.2014.10.016, 2015.

Anderson, P. M., Lozhkin, A. V., Belaya, B. V., and Stetsenko, T. V.: New data about the stratigraphy of late Quaternary deposits of northern Priokhot'ye in: Environmental changes in Beringia during the Quaternary, edited by: Simakov, K. V., North East Interdisciplinary Research Institute, Far East Branch, Russian Academy of Sciences, Magadan, 69-87, 1998a (in Russian).

Anderson, P. M., Lozhkin, A. V. and Belaya, B. V.: Younger Dryas in western Beringia (northeastern Siberia), in: Environmental changes in Beringia during the Quaternary, North East Interdisciplinary Research Institute, edited by: Simakov, K. V., Far East
Branch, Russian Academy of Sciences, Magadan, 28-44, 1998b (in Russian).

Anderson, P. M., Lozhkin, A. V., Solomatkina, T. B., and Brown, T. A.: Paleoclimatic implications of glacial and postglacial refugia for Pinus pumila in western Beringia, Quaternary Res., 73, 269276, https://doi.org/10.1016/j.yqres.2009.09.008, 2010.

Andreev, A. A. and Klimanov, V. A.: Vegetation and climate history of central Yakutia during Holocene and late Pleistocene, in: Formirovanie rel'efa, korrelyatnykh otlozhenii i rossypei severovostoka SSSR (Formation of deposits and placers on north-east of the USSR), Magadan, Russia, 26-51, 1989.

Andreev, A. A. and Klimanov, V. A.: Vegetation History and climate changes in the interfluve of the Rivers Ungra and Yakokit (the southern Yakutia) in Holocene, Botanichesky Zhurnal (Botanical Journal), 76, 334-351, 1991.

Andreev, A. A. and Klimanov, V. A.: Quantitative Holocene climatic reconstruction from Arctic Russia, J. Paleolimnol., 24, 8191, https://doi.org/10.1023/a:1008121917521, 2000.

Andreev, A. A., Klimanov, V. A., Sulerzhitskii, L. D., and Khotinskii, N. A.: Chronology of environmental changes in central Yakutia during the Holocene, In: Paleoklimaty golotsena i pozdnelednikov'ya (Paleoclimates of Holocene and late glacial), Nauka, Moscow, Russia, 115-121, 1989.

Andreev, A. A., Manley, W. F., Ingólfsson, Ó., and Forman, S. L.: Environmental changes on Yugorski Peninsula, Kara Sea, Russia, during the last 12,800 radiocarbon years, Global Planet. Change, 31, 255-264, https://doi.org/10.1016/S0921-8181(01)00123-0, 2001.

Andreev, A. A., Schirrmeister, L., Siegert, C., Bobrov, A. A., Demske, D., Seiffert, M., and Hubberten, H.-W.: Paleoenvironmental changes in northeastern Siberia during the Late Quaternary - evidence from pollen records of the Bykovsky Peninsula, Polarforschung, 70, 13-25, 2002.

Andreev, A. A., Tarasov, P. E., Siegert, C., Ebel, T., Klimanov, V. A., Melles, M., Bobrov, A. A, Dereviagin, A. Y., Lubinski, D. J., and Hubberten, H.-W.: Late Pleistocene and Holocene vegetation and climate on the northern Taymyr Peninsula, Arctic Russia, Boreas, 32, 484-505, https://doi.org/10.1111/j.15023885.2003.tb01230.x, 2003.

Andreev, A. A., Tarasov, P. E., Schwamborn, G., Ilyashuk, B. P., Ilyashuk, E. A., Bobrov, A. A., Klimanov, V. A., Rachold, V., and Hubberten, H.-W.: Holocene paleoenvironmental records from Nikolay Lake, Lena River Delta, Arctic Russia, Palaeogeogr. Palaeoecol., 209, 197-217, https://doi.org/10.1016/j.palaeo.2004.02.010, 2004.

Andreev, A. A., Tarasov, P. E., Ilyashuk, B. P., Ilyashuk, E. A., Cremer, H., Hermichen, W. D., Wischer, F., and Hubberten, H.W.: Holocene environmental history recorded in Lake Lyadhej To sediments, Polar Urals, Russia, Palaeogeogr. Palaeocl., 223, 181-203, https://doi.org/10.1016/j.palaeo.2005.04.004, 2005.

Andreev, A. A., Morozova, E., Fedorov, G., Schirrmeister, L., Bobrov, A. A., Kienast, F., and Schwamborn, G.: Vegetation history of central Chukotka deduced from permafrost paleoenvironmental records of the El'gygytgyn Impact Crater, Clim. Past, 8, 1287-1300, https://doi.org/10.5194/cp-8-1287-2012, 2012.

Arkhipov, S. A. and Votakh, M. R.: Palynological characteristics and the absolute age of peat near the mouth of the Tom' River, in: The palynology of Siberia, edited by: Saks, V. N., Nauka, Moscow, 112-118, 1980 (in Russian). 
Bakhareva, V. A.: Palynological characteristics of sediments of the second floodplain terrace of the lower Irtysh River, in: Glaciation and paleoclimates of Siberia during the Pleistocene, edited by: Arkhipov, S. A., Volkova, V. S., and Skabichevskaya, N. A., Institute of Geology and Geophysics, Siberian Branch, USSR Academy of Sciences, Novosibirsk, 79-82, 1983 (in Russian).

Bazarova, V. B., Klimin, M. A., Mokhova, L. M., and Orlova, L. A.: New pollen records of Late Pleistocene and Holocene changes of environment and climate in the Lower Amur River basin, NE Eurasia, Quatern. Int., 179, 9-19, https://doi.org/10.1016/j.quaint.2007.08.015, 2008.

Bezrukova, E. V., Belov, A. V., Abzaeva, A. A., Letunova, P. P., Orlova, L. A., Sokolova, L. P., Kulagina, N. V., and Fisher, E. E.: First High-Resolution Dated Records of Vegetation and Climate Changes on the Lake Baikal Northern Shore in the Middle-Late Holocene, Dokl. Earth Sci., 411, 1331-1335, https://doi.org/10.1134/S1028334X0608037X, 2006.

Bezrukova, E. V., Belov, A. V., and Orlova, L. A.: Holocene vegetation and climate variability in North Pre-Baikal region, East Siberia, Russia, Quatern. Int., 237, 74-82, https://doi.org/10.1016/j.quaint.2011.01.012, 2011.

Bigelow, N. H., Brubaker, L. B., Edwards, M. E., Harrison, S. P., Prentice, I. C., Anderson, P. M., Andreev, A. A., Bartlein, P. J., Christensen, T. R., Cramer, W., Kaplan, J. O., Lozhkin, A. V., Matveyeva, N. V., Murray, D. F., McGuire, A. D., Razzhivin, V. Y., Ritchie, J. C., Smith, B., Walker, D. A., Gajewski, K., Wolf, V., Holmqvist, B. H., Igarashi, Y., Kremenetskii, K., Paus, A., Pisaric, M. F. J., and Volkova, V. S.: Climate change and arctic ecosystems: 1 . Vegetation changes north of $55^{\circ} \mathrm{N}$ between the last glacial maximum, mid-Holocene, and present, J. Geophys. Res., 108, 8170, https://doi.org/10.1029/2002JD002558, 2003.

Bigelow, N. H., Brubaker, L. B., Edwards, M. E., Harrison, S. P., Prentice, I. C., Anderson, P. M., Andreev, A. A., Bartlein, P. J., Christensen, T. R., Cramer, W., Kaplan, J. O., Lozhkin, A. V., Matveyeva, N. V., Murray, D. F., McGuire, A. D., Razzhivin, V. Y., Ritchie, J. C., Smith, B., Walker, D. A., Gajewski, K., Wolf, V., Holmqvist, B. H., Igarashi, Y., Kremenetskii, K., Paus, A., Pisaric, M. F. J., and Volkova, V. S.: Climate change and arctic ecosystems: 1. Vegetation changes north of $55^{\circ} \mathrm{N}$ between the last glacial maximum, mid-Holocene, and present, J. Geophys. Res., 108, 8170, https://doi.org/10.1029/2002JD002558, 2008.

Binney, H., Edwards, M. E., Macias-Fauria, M., Lozhkin, A., Anderson, P., Kaplan, J. O., Andreev, A., Bezrukova, E., Blyakharchuk, T., Jankovsha, V., Khazina, I., Krivonogov, S., Kremenetski, K., Nield, J., Novenko, E., Ryabogina, N., Solovieva, N., Willis, K. J., and Zernitskaya, V.: Vegetation of Eurasia from the last glacial maximum to present: key biogeographic patterns, Quaternary Sci. Rev., 157, 80-97, https://doi.org/10.1016/j.quascirev.2016.11.022, 2017.

Binney, H. A., Willis, K. J., Edwards, M. E., Bhagwat, S. A., Anderson, P. M., Andreev, A. A., Blaauw, M., Damblon, F., Haesaerts, P., Kienast, F., Kremenetski, K. V., Krivonogov, S. K., Lozhkin, A. V., MacDonald, G. M., Novenko, E. Y., Oksanen, P., Sapelko, T. V., Väliranta, M., and Vazhenina, L.: The distribution of late-Quaternary woody taxa in northern Eurasia: evidence from a new macrofossil database, Quaternary Sci. Rev., 28, 2445-2464, https://doi.org/10.1016/j.quascirev.2009.04.016, 2009.
Biskaborn, B. K., Subetto, D. A., Savelieva, L. A., Vakhrameeva, P. S., Hansche, A., Herzschuh, U., Klemm, J., Heinecke, L., Pestryakova, L. A., Meyer, H., Kuhn, G., and Diekmann, B.: Late Quaternary vegetation and lake system dynamics in north-eastern Siberia: implications for seasonal climate variability, Quaternary Sci. Rev., 147, 406-421, https://doi.org/10.1016/j.quascirev.2015.08.014, 2016.

Blaauw, M. and Christen, J. A.: Flexible paleoclimate age-depth models using an autoregressive gamma process, Bayesian Anal., 6, 457-474, 2011.

Blyakharchuk, T. A.: Istorija rastitel'nosti yugo-vostoka Zapadnoi Sibiri $\mathrm{v}$ golotsene po dannym botanicheskogo I sporovopyl'tsevogo analiza torfa (The Holocene history of vegetation of south-eastern West Siberia by botanical and pollen analyses of peat deposits), PhD thesis, Tomsk State University, Tomsk, Russia, 248 pp., 1989.

Blyakharchuk, T. A.: Four new pollen sections tracing the Holocene vegetational development of the southern part of the West Siberian Lowland, Holocene, 13, 715-731, https://doi.org/10.1191/0959683603hl658rp, 2003.

Borisova, O. K., Novenko, E. Y., Zelikson, E. M., and Kremenetski, K. V.: Lateglacial and Holocene vegetational and climatic changes in the southern taiga zone of West Siberia according to pollen records from Zhukovskoye peat mire, Quatern. Int., 237, 65-73, https://doi.org/10.1016/j.quaint.2011.01.015, 2011.

Cao, X., Ni, J., Herzschuh, U., Wang, Y., and Zhao, Y.: A late Quaternary pollen dataset from eastern continental Asia for vegetation and climate reconstructions: set up and evaluation, Rev. Palaeobot. Palyno., 194, 21-37, https://doi.org/10.1016/j.revpalbo.2013.02.003, 2013.

Cao, X., Herzschuh, U., Ni, J., Zhao, Y., and Böhmer, T.: Spatial and temporal distributions of major tree taxa in eastern continental Asia during the last 22,000 years, Holocene, 25, 79-91, https://doi.org/10.1111/oik.01525, 2015.

Cao, X., Tian, F., Andreev, A., Anderson, P. M., Lozhkin, A. V., Bezrukova, E. V., Ni, J., Rudaya, N., Stobbe, A., and Herzschuh, U.: A taxonomically harmonized and temporally standardized fossil pollen dataset from Siberia covering the last $40 \mathrm{ka}, \mathrm{PAN}-$ GAEA, https://doi.org/10.1594/PANGAEA.898616, 2019a.

Cao, X., Tian, F., Li, F., Gaillard, M.-J., Rudaya, N., Xu, Q., and Herzschuh, U.: Pollen-based quantitative land-cover reconstruction for northern Asia covering the last $40 \mathrm{kacal} \mathrm{BP}$, Clim. Past, 15, 1503-1536, https://doi.org/10.5194/cp-15-1503-2019, 2019b.

Demske, D., Heumann, G., Granoszewski, W., Nita, M., Mamakowa, K., Tarasov, P. E., and Oberhansli, H.: Late glacial and Holocene vegetation and regional climate variability evidenced in high-resolution pollen records from Lake Baikal, Global Planet. Change, 46, 255-279, https://doi.org/10.1016/j.gloplacha.2004.09.020, 2005.

Dirksen, V., Dirksen, O., Christel, V. D. B., and Diekmann, B.: Holocene pollen record from Lake Sokoch, interior Kamchatka (Russia), and its paleobotanical and paleoclimatic interpretation, Global Planet. Change, 58, 46-47, https://doi.org/10.1016/j.gloplacha.2015.07.010, 2012.

Firsov, L. V., Levina, T. P., and Troitskii, S. L.: The Holocene climatic changes in northern Siberia, in: Climatic changes in arctic areas during the last ten thousand years, Acta Universitatis Onlensis, Section A, edited by: Vasari, V., Hyvarinen, H. and Hicks, 
S., Scientitae Rerum Naturalium Number 3, Geologica Number 3, University of Oula, Oula, 341-349, 1972.

Gaillard, M.-J., Sugita, S., Mazier, F., Trondman, A.-K., Broström, A., Hickler, T., Kaplan, J. O., Kjellström, E., Kokfelt, U., Kuneš, P., Lemmen, C., Miller, P., Olofsson, J., Poska, A., Rundgren, M., Smith, B., Strandberg, G., Fyfe, R., Nielsen, A. B., Alenius, T., Balakauskas, L., Barnekow, L., Birks, H. J. B., Bjune, A., Björkman, L., Giesecke, T., Hjelle, K., Kalnina, L., Kangur, M., van der Knaap, W. O., Koff, T., Lagerås, P., Latałowa, M., Leydet, M., Lechterbeck, J., Lindbladh, M., Odgaard, B., Peglar, S., Segerström, U., von Stedingk, H., and Seppä, H.: Holocene landcover reconstructions for studies on land cover-climate feedbacks, Clim. Past, 6, 483-499, https://doi.org/10.5194/cp-6-4832010, 2010.

Gajewski, K.: The global pollen database in biogeographical and palaeoclimatic studies, Prog. Phys. Geog., 32, 379-402, https://doi.org/10.1177/0309133308096029, 2008.

Hoff, U., Biskaborn, B. K., Dirksen, V. G., Dirksen, O. V., Kuhn, G., Meyer, H., Nazarova, L. B., Roth, A., and Diekmann, B.: Holocene environment of Central Kamchatka, Russia: Implications from a multi-proxy record of Two-Yurts Lake, Global Planet. Change, 134, 101-117, https://doi.org/10.1016/j.gloplacha.2015.07.011, 2015.

Ivanov, V. F.: Quaternary deposits of the coast of eastern Chukotka, North East Interdisciplinary Research Institute, Far East Branch, USSR Academy of Sciences, Vladivostok, 1986 (in Russian).

Ivanov, V. F., Lozhkin, A. V., Kal'nichenko, S. S., Kyshtymov, A. I., Narkhinova, V. E., and Terekhova, V. E.: The late Pleistocene and Holocene of Chukchi Peninsula to the north of Kamchatka, in: Geology and useful minerals of northeast Asia, edited by: Goncharov, V. I., Far East Branch, USSR Academy of Sciences, Vladivostok, 33-42, 1984 (in Russian).

Jankovská, V., Andreev, A. A., and Panova, N. K.: Holocene environmental history on the eastern slope of the Polar Ural Mountains, Russia, Boreas, 35, 650-661, 2006.

Juggins, S.: rioja: Analysis of Quaternary Science Data, version 0.7-3, available at: http://cran.r-project.org/web/packages/rioja/ index.html (last access: October 2016), 2012.

Kats, S. V.: History of vegetation of western Siberia during the Holocene, Bulletin of commission for study the Quaternary, 13, 118-123, Nauka, Moscow, 1953 (in Russian).

Khomutova, V. and Pushenko, M,: Evolution of lake ecosystem of Southern Ural (Russia) from palynological data, Abstract of 14 Symposium "Palynologie \& changements globaux", Paris, 1995.

Klemm, J., Herzschuh, U., and Pestryakova, L. A.: Vegetation, climate and lake changes over the last 7,000 years at the boreal treeline in north-central Siberia, Quaternary Sci. Rev., 147, 422-434, https://doi.org/10.1016/j.quascirev.2015.08.015, 2016.

Korotky, A. M.: Paleographic conditions of the formation of Quaternary peats (south of Far East), in: Modern sedimentation and morpholithogenesis of the Far East, edited by: Pletnev, S. P. and Pushkar, V. S., Far Eastern Science Center, USSR Academy of Sciences, Vladivostok, 58-71, 1982 (in Russian).

Korotky, A. M. and Karaulova, L. P.: New data about the stratigraphy of Quaternary deposits of Primor'ye, in: Questions of geomorphology and Quaternary geology of the southern Far East, edited by: Korotky, A. M. and Kulakov, A. P., Far Eastern Branch, USSR Academy of Sciences, Vladivostok, 79-110, 1975 (in Russian).
Korotky, A. M. and Kovalyukh, N. N.: Rhythms of perrenial permafrost: a reflection of climate changes during the late Pleistocene and Holocene, in: Paleogeographic investigations in the Far East, edited by: Korotky, A. M. and Pushkar, V. S., Far Eastern Science Center of the USSR Academy of Sciences, Vladivostok, 20-36, 1987 (in Russian).

Korotky, A. M., Karaulova, L. P., and Troitskaya, T. S.: The Quaternary deposits of Primor'ye, Nauka, Novosibirsk, 1980 (in Russian).

Korotky, A. M., Pletnev, S. P., Pushkar, V. S., Grebennikova, T. A., Razzhigaeva, N. G., Sakhebgareeva, E. D., and Mokhova, L. M.: Evolution environment of south Far East (late Pleistocene and Holocene), Nauka, Moscow, 1988 (in Russian).

Korotky, A. M., Mokhova, L. M., and Pushkar, V. S.: The climate changes of the Holocene and landscape evolution of bald mountains of central Yam-Alin', in: Paleogeographic investigations in the Far East, edited by: Korotky, A. M. and Pushkar, V. S., Far Eastern Science Center of the USSR Academy of Sciences, Vladivostok, 5-22, 1985 (in Russian).

Korotky, A. M., Volkov, V. G., Bazarova, V. B., and Kovalyukh, N. N.: The catalog of radiocarbon dates of Quaternary deposits of the Far East, Far Eastern Branch, Russian Academy of Sciences, Vladivostok, 1993 (in Russian).

Korotky, A. M., Razzhigaeva, N. G., Grebennikova, T. A., Ganzey, L. A., Mokhova, L. M., Bazarova, V. B., and Sulerzhitsky, L. D.: Holocene marine terraces of Kunashir Island, Kuril Islands. Quaternary Res., 34, 359-375, 1995.

Korotky, A. M., Razzhigaeva, N. G., Mokhova, L. M., Ganzey, L. A., Grebennikova, T. A., and Bazarova, V. B.: Coastal dunes as an indicator of period of global climatic deterioration (Kunashir Island, Kuriles), Geol. Pac. Ocean, 13, 73-84, 1996.

Korotky, A. M., Grebennikova, T. A., Razzhigaeva, N. G., Volkov, V. G., Mokhova, L. M., Ganzey, L. A., and Bazarova, V. B.: Marine terraces of western Sakhalin Island, Catena, 30, 61-81, https://doi.org/10.1016/S0341-8162(97)00002-7, 1997a.

Korotky, A. M., Pushkar, V. S., Grebennikova, T. A., Razzhigaeva, N. G., Karaulova, L. P., Mokhova, L. M., Ganzey, L. A., Cherepanova, M. N., Bazarova, V. B., Volkov, V. G., and Kovalyukh, N. N.: Marine terraces and Quaternary history of the Sakhalin Shelf, Dal'nauka, Vladivostok, 1997b (in Russian).

Kraus, M., Matthiessen, J., and Stein, R.: A Holocene marine pollen record from the northern Yenisei Estuary (southeastern Kara Sea, Siberia), in: Siberian River Run-off in the Kara Sea: Characterisation, Quantification, Variability, and Environmental Significance, edited by: Stein, R., Fahl, K., Fütterer, D. K., Galimov, E. M., and Stepanets, O. V., Proceedings in Marine Sciences, Elsevier, Amsterdam, 6, 433-456, https://doi.org/10.1016/S15682692(03)80048-7, 2003.

Kremenetski, C. V., Bottger, T., Junge, F. W., and Tarasov, A. G.: Late- and postglacial environment of the Buzuluk area, middle Volga region, Russia, Quaternary Sci. Rev., 18, 1185-1203, https://doi.org/10.1016/S0277-3791(98)00074-2, 1999.

Kremenetskii, K. V., Tarasov, P. E., and Cherkinski, A. E.: Istoriva ostrovnykh borov Kazakhstana $\mathrm{v}$ golotsene (Holocene history of the Kazakhstan "island” pine forests), Botanicheski Zhurial (Botanical Journal), 79, 13-29, 1994.

Leipe, C., Nakagawa, T., Gotanda, K., Müller, S., and Tarasov, P. E.: Late Quaternary vegetation and climate dynamics at the northern limit of the East Asian summer monsoon and its re- 
gional and global-scale controls, Quaternary Sci. Rev., 116, 5771, https://doi.org/10.1016/j.quascirev.2015.03.012, 2015.

Lisitsyna, O. V., Giesecke, T., and Hicks, S.: Exploring pollen percentage threshold values as an indication for the regional presence of major European trees, Rev. Palaeobot. Palyno., 166, 311324, https://doi.org/10.1016/j.revpalbo.2011.06.004, 2011.

López-García, P., López-Sáez, J. A., Chernykh, E. N., and Tarasov, P. E.: Late Holocene vegetation history and human activity shown by pollen analysis of Novienki peat bog (Kargaly region, Orenburg Oblast, Russia), Veg. Hist. Archaeobot., 12, 75-82, https://doi.org/10.1007/s00334-003-0009-4, 2003.

Lozhkin, A. V.: Questions concerning radiocarbon data and palynological characteristics of the mammoth burials, Berelyekh River, lower Indigirka drainage, in: Environmental changes in Beringia during the Quaternary, North East Interdisciplinary Research Institute, edited by: Simakov, K. V., Far East Branch, Russian Academy of Sciences, Magadan, 45-62, 1998 (in Russian).

Lozhkin, A. and Anderson, P.: Late Quaternary lake records from the Anadyr Lowland, Central Chukotka (Russia), Quaternary Sci. Rev., 68, 1-16, https://doi.org/10.1016/j.quascirev.2013.02.007, 2013.

Lozhkin, A. V. and Glushkova, O. Y.: New palynological assemblages and radiocarbon dates from the late Quaternary deposits of northern Priokhot'ye in: Late Pleistocene and Holocene of Beringia, edited by: Gagiev, M. K., North East Interdisciplinary Research Institute, Far East Branch, Russian Academy of Sciences, Magadan, 70-79, 1997a (in Russian).

Lozhkin, A. V. and Glushkova, O. Y.: Boreal peats in the upper Kolyma basin in: Late Pleistocene and Holocene of Beringia, edited by: Gagiev, M. K., North East Interdisciplinary Research Institute, Far East Branch, Russian Academy of Sciences, Magadan, 55-62, 1997b (in Russian).

Lozhkin, A. V. and Postolenko, G. A.: New data about the environmental evolution of the mountain region of the Kolyma region during the late Anthropogene, Dokl. Akad. Nauk, 307, 11841188, 1989.

Lozhkin, A. V. and Prokhorova, T. P.: New data about the history of alas deposits from the Kolyma lowland (radiocarbon and palynological material), in: Stratigraphy and paleogeography of the late Cenozoic of eastern USSR, edited by: Biske, S. F., North East Interdisciplinary Research Institute, Far East Branch, USSR Academy of Sciences, Magadan, 96-102, 1982 (in Russian).

Lozhkin, A. V. and Vazhenina, L. N.: The characteristics of vegetational development from the Kolyma lowland in the early Holocene, in: Quaternary period of northeast Asia, edited by: Pokhialainen, V. P., North East Interdisciplinary Research Institute, Far East Branch, USSR Academy of Sciences, Magadan, 135-144, 1987 (in Russian).

Lozhkin, A. V., Prokhorova, T. P., and Parii, V. P.: Radiocarbon dates and palynological characteristics of sediments of the alas complex of the Kolyma Lowland, Dokl. Akad. Nauk, 224, 13951398, 1975 (in Russian).

Lozhkin, A. V., Skorodumov, I. N., Meshkov, A. P., and Rovako, L. G.: Changed paleogeographic environments in the region of Glukhoye Lake (north coast of the Okhotsk Sea) during the Pleistocene-Holocene transition, Dokl. Akad. Nauk, 316, 184 188, 1990 (in Russian).

Lozhkin, A. V., Anderson, P. M., Eisner, W. R., Ravako, L. G., Hopkins, D. M., Brubaker, L. B., Colinvaux, P. A., and Miller, M. C.: Late Quaternary lacustrine pollen records from southwestern Beringia, Quaternary Res., 39, 314-324, https://doi.org/10.1006/qres.1993.1038, 1993.

Lozhkin, A. V., Anderson, P. M., Belaya, B. V., Glushkova, O. Y., and Stetsenko, T. V.: Vegetation change in northeast Siberia at the Pleistocene-Holocene boundary and during the Holocene, in: The Quaternary period of Beringia, edited by: Simakov, K. V., North East Interdisciplinary Research Institute, Far East Branch, Russian Academy of Sciences, Magadan, 53-75, 2000a (in Russian).

Lozhkin, A. V., Anderson, P. M., Belaya, B. V., Glushkova, O. Y., Kotova, L. N., and Trumpe, M. A.: Palynological characteristics and radiocarbon dates from late Holocene lacustrine sediments of northern Priokhot'ye, in: The Quaternary period of Beringia, edited by: Simakov, K. V., North East Interdisciplinary Research Institute, Far East Branch, Russian Academy of Sciences, Magadan, 76-87, 2000b (in Russian).

Lozhkin, A. V., Kotov, A. N., and Ryabchun, V. K.: Palynological and radiocarbon data of the Ledovyi Obryv exposure (the south east of Chukotka), in: The Quaternary Period of Beringia, North East Interdisciplinary Research Institute, edited by: Simakov, K. V., Far East Branch, Russian Academy of Sciences, Magadan, 118-131, 2000c (in Russian).

Melles, M., Brigham-Grette, J., Minyuk, P. S., Nowaczyk, N. R., Wennrich, V., DeConto, R. M., Anderson, P. A., Andreev, A. A., Coletti, A., Cook, T. L., Haltia-Hovi, E., Kukkonen, M., Lozhkin, A. V., Rosén, P., Tarasov, P .E., Vogel, H., and Wagner, B.: 2.8 Million years of Arctic climate change from Lake El'gygytgyn, NE Russia, Science, 337, 315-320, https://doi.org/10.1126/science.1222135, 2012.

Neustadt, M. I. and Zelikson, E. M.: Neue Angaben zur stratigraphie der Torfmoore Westsibiriens, Acta Agralia Fennica, 123, 27-32, 1985.

Magri, D.: Patterns of post-glacial spread and the extent of glacial refugia of European beech (Fagus syvatica), J. Biogeogr., 35, 450-463, https://doi.org/10.1111/j.1365-2699.2007.01803.x, 2008.

Marsicek, J., Shuman, B. N., Bartlein, P., Shafer, S. L., and Brewer, S.: Reconciling divergent trends and millennial variations in Holocene temperatures, Nature, 554, 92-96, https://doi.org/10.1038/nature25464, 2018.

Mauri, A., Davis, B. A. S., Collins, P. M., and Kaplan, J. O.: The climate of Europe during the Holocene: a gridded pollen-based reconstruction and its multiproxy evaluation, Quaternary Sci. Rev., 112, 109-127, https://doi.org/10.1016/j.quascirev.2015.01.013, 2015.

Mokhova, L., Tarasov, P., Bazarova, V., and Klimin, M.: Quantitative biome reconstruction using modern and late Quaternary pollen data from the southern part of the Russian Far East, Quaternary Sci. Rev., 28, 2913-2926, https://doi.org/10.1016/j.quascirev.2009.07.018, 2009.

Müller, S., Tarasov, P. E., Andreev, A. A., and Diekmann, B.: Late Glacial to Holocene environments in the present-day coldest region of the Northern Hemisphere inferred from a pollen record of Lake Billyakh, Verkhoyansk Mts, NE Siberia, Clim. Past, 5, 73-84, https://doi.org/10.5194/cp-5-73-2009, 2009.

Müller, S., Tarasov, P. E., Andreev, A. A., Tütken, T., Gartz, S., and Diekmann, B.: Late Quaternary vegetation and environments in the Verkhoyansk Mountains region 
(NE Asia) reconstructed from a 50-kyr fossil pollen record from Lake Billyakh, Quaternary Sci. Rev., 29, 2071-2086, https://doi.org/10.1016/j.quascirev.2010.04.024, 2010.

Naidina, O. D. and Bauch, H. A.: A Holocene pollen record from the Laptev Sea shelf, northern Yakutia, Global Planet. Change, 31, 141-153, https://doi.org/10.1016/S0921-8181(01)00117-5, 2001.

Neishtadt, M. I. (Ed.): Holocene processes in western Siberia and associated problems, in: Studying and mastering the environment, USSR Academy of Sciences, Institute of Geography, Moscow, 90-99, 1976a (in Russian).

Neishtadt, M. I.: About environmental changes in middle taiga of western Siberia during the Holocene, in: Palynology of the USSR, edited by: Zaklinskaya, E. D. and Neishtadt, M. I., Nauka, Moscow, 156-161, 1976b (in Russian).

Ni, J., Cao, X., Jeltsch, F., and Herzschuh, U.: Biome distribution over the last 22,000 yr in China, Palaeogeogr. Palaeocl., 409, $33-$ 47, https://doi.org/10.1016/j.palaeo.2014.04.023, 2014.

Niemeyer, B., Klemm, J., Pestryakova, J. A., and Herzschuh, U.: Relative pollen productivity estimates for common taxa of the northern Siberian Arctic, Rev. Palaeobot. Palyno., 221, 71-82, https://doi.org/10.1016/j.revpalbo.2015.06.008, 2015.

Oganesyan, A. S., Prokhorova, T. P., Trumpe, M. A., and Susekova, N. G.: Paleosols and peats of Wrangel Island, Pochvovedenie, 2, 15-28, 1993 (in Russian).

Panova, N.: Novye dannye po paleoekologii i istorii rastitelnosti yuzhnogo Yamala v golotsene (New data for paleoecology and vegetation history of southern Yamal during the Holocene), in: Chetvertichnyi period: metody issledovania, strat, 45-46, 1990.

Panova, N.: Palinologicheskoe issledovanie Karasieozerskogo torfyanika na srednem Urale (Palynological study of Karasieozerskiy peatland on Middle Ural), in: Issledovanie lesov Urala. Materialy nauchnykh chteniy posvyaschennykh pamyati B, 2831, 1997.

Panova, N., Makovsky, V. I., and Erokhin, N. G.: Golotsenovaya dinamika rastitelnosti $\mathrm{v}$ raione Krasnoufimskoi stepi (Holocene dynamics of vegetation in Krasnoufimskaya forest-steppe area), in: Lesoobrazovatelnyi protses na Urale i v Zaurali, 80-93, 1996.

Peteet, D. M., Andreev, A. A., Bardeen, W., and Mistretta, F.: Longterm Arctic peatland dynamics, vegetation and climate history of the Pur-Taz region, Western Siberia, Boreas, 27, 115-126, https://doi.org/10.1111/j.1502-3885.1998.tb00872.x, 1998.

Pisaric, M. F. J., MacDonald, G. M., Velichko, A. A., and Cwynar, L. C.: The Lateglacial and Postglacial vegetation history of the northwestern limits of Beringia, based on pollen, stomate and tree stump evidence, Quaternary Sci. Rev., 20, 235-245, https://doi.org/10.1016/S0277-3791(00)00120-7, 2001.

Pitkänen, A., Turunen, J., Tahvanainen, T., and Tolonen, K.: Holocene vegetation history from the SalymYugan Mire Area, West Siberia, Holocene, 12, 353-362, https://doi.org/10.1191/0959683602hl533rp, 2002.

Rudaya, N., Nazarova, L., Nourgaliev, D., Palagushkina, O., Papin, D., and Frolova, L.: Mid-late Holocene environmental history of Kulunda, southern West Siberia: vegetation, climate and humans, Quaternary Sci. Rev. 48, 32-42, https://doi.org/10.1016/j.quascirev.2012.06.002, 2012.

Sánchez Goñi, M. F., Desprat, S., Daniau, A.-L., Bassinot, F. C., Polanco-Martínez, J. M., Harrison, S. P., Allen, J. R. M., Anderson, R. S., Behling, H., Bonnefille, R., Burjachs, F., Carrión, J. S.,
Cheddadi, R., Clark, J. S., Combourieu-Nebout, N., Mustaphi, Colin. J. Courtney, Debusk, G. H., Dupont, L. M., Finch, J. M., Fletcher, W. J., Giardini, M., González, C., Gosling, W. D., Grigg, L. D., Grimm, E. C., Hayashi, R., Helmens, K., Heusser, L. E., Hill, T., Hope, G., Huntley, B., Igarashi, Y., Irino, T., Jacobs, B., Jiménez-Moreno, G., Kawai, S., Kershaw, A. P., Kumon, F., Lawson, I. T., Ledru, M.-P., Lézine, A.-M., Liew, P. M., Magri, D., Marchant, R., Margari, V., Mayle, F. E., McKenzie, G. M., Moss, P., Müller, S., Müller, U. C., Naughton, F., Newnham, R. M., Oba, T., Pérez-Obiol, R., Pini, R., Ravazzi, C., Roucoux, K. H., Rucina, S. M., Scott, L., Takahara, H., Tzedakis, P. C., Urrego, D. H., van Geel, B., Valencia, B. G., Vandergoes, M. J., Vincens, A., Whitlock, C. L., Willard, D. A., and Yamamoto, M.: The ACER pollen and charcoal database: a global resource to document vegetation and fire response to abrupt climate changes during the last glacial period, Earth Syst. Sci. Data, 9, 679-695, https://doi.org/10.5194/essd-9-679-2017, 2017.

Shichi, K., Takahara, H., Krivonogovc, S. K., Bezrukova, E. V., Kashiwaya, K., Takehara, A., and Nakamura, T.: Late Pleistocene and Holocene vegetation and climate records from Lake Kotokel, central Baikal region, Quatern. Int., 205, 98-110, https://doi.org/10.1016/j.quaint.2009.02.005, 2009.

Shilo, N. A., Lozhkin, A. V., Titov, E. E., and Schumilov, Y. V.: Kirgirlakh mammoth: paleography aspect, Nauka, Moscow, 1983 (in Russian).

Stetsenko, T. V.: A pollen record from Holocene Lake deposits in the Malyk-Siena depression, upper Kolyma basin, in: Environmental changes in Beringia during the Quaternary, edited by: Simakov, K. V., North East Interdisciplinary Research Institute, Far East Branch, Russian Academy of Sciences, Magadan, 6368, 1998 (in Russian).

Stobbe, A., Gumnior, M., Roepke, A., and Schneider, H.: Palynological and sedimentological evidence from the TransUral steppe (Russia) and its palaeoecological implications for the sudden emergence of Bronze Age sedentarism, Veg. Hist. Archaeobot., 24, 393-412, https://doi.org/10.1007/s00334-0140500-0, 2015.

Sugita, S., Parshall, T., Calcote, R., and Walker, K.: Testing the Landscape Reconstruction Algorithm for spatially explicit reconstruction of vegetation in northern Michigan and Wisconsin, Quaternary Res., 74, 289-300, https://doi.org/10.1016/j.yqres.2010.07.008, 2010.

Tarasov, P. E., Webb, T., Andreev, A. A, Afanas'Eva, N. B., Berezina, N. A., Bezusko, L. G., Blyakharchuk, T. A., Bolikhovskaya, N. S., Cheddadi, R., Chernavskaya, M. M., Chernova, G. M., Dorofeyuk, N. I., Dirksen, V. G., Elina, G. A., Filimonova, L. V., Glebov, F. Z., Guiot, J., Gunova, V. S., Harrison, S. P., Jolly, D., Khomutova, V. I., Kvavadze, E. V., Osipova, I. M., Panova, N. K., Prentice, I. C., Saarse, L., Sevastyanov, D. V., Volkova, V. S., and Zernitskaya, V. P.: Present-day and mid-Holocene biomes reconstructed from pollen and plant macrofossil data from the Former Soviet Union and Mongolia, J. Biogeogr., 25, 1029-1053, https://doi.org/10.1046/j.1365-2699.1998.00236.x, 1998.

Tarasov, P. E., Volkova, V. S., Webb, T., Guiot, J., Andreev, A. A., Bezusko, L. G., Bezusko, T. V., Bykova, G. V., Dorofeyuk, N. I., Kvavadze, E. V., Osipova, I. M., Panova, N. K., and Sevastyanov, D. V.: Last glacial maximum biomes reconstructed from pollen and plant macrofossil data from northern Eurasia, J. Biogeogr., 
27, 609-620, https://doi.org/10.1046/j.1365-2699.2000.00429.x, 2000.

Tarasov, P. E., Williams, J. W., Andreev, A., Nakagawa, T., Bezrukova, E., Herzschuh, U., Igarashi, Y., Müller, S., Werner, K., and Zheng, Z.: Satellite- and pollen-based quantitative woody cover reconstructions for northern Asia: Verification and application to late-Quaternary pollen data, Earth Planet. Sc. Lett., 264, 284-298, https://doi.org/10.1016/j.eps1.2007.10.007, 2007.

Tarasov, P. E., Andreev, A. A., Romanenko, F. A., and Sulerzhitskii, L. D.: Palynostratigraphy of upper Quaternary deposits of Sverdrup Island, the Kara Sea, Stratigr. Geol. Correl., 3, 190-196, https://doi.org/10.1016/0037-0738(94)00119-F, 1995.

Tarasov, P. E., Nakagawa, T., Demske, D., Österle, H., Igarashi, Y., Kitagawa, J., Mokhova, L. M., Bazarova, V. B., Okuda, M., Gotanda, K., Miyoshi, N., Fujiki, T., Takemura, K., Yonenobu, H., and Fleck, A.: Progress in the reconstruction of Quaternary climate dynamics in the Northwest Pacific: A new modern analogue reference dataset and its application to the 430kyr pollen record from Lake Biwa, Earth-Sci. Rev., 108, 64-79, https://doi.org/10.1016/j.earscirev.2011.06.002, 2011.

Tian, F., Cao, X., Dallmeyer, A., Ni, J., Zhao, Y., Wang, Y., and Herzschuh, U.: Quantitative woody cover reconstructions from eastern continental Asia of the last $22 \mathrm{ka}$ reveal strong regional peculiarities, Quaternary Sci. Rev., 137, 33-44, https://doi.org/10.1016/j.quascirev.2016.02.001, 2016.

Tian, F., Cao, X., Dallmeyer, A., Lohmann, G., Zhang, X., Ni, J., Andreev, A., Anderson, P. M., Lozhkin, A. V., Bezrukova, E., Rudaya, N., Xu, Q., and Herzschuh, U.: Biome changes and their inferred climatic drivers in northern and eastern continental Asia at selected times since $40 \mathrm{cal} \mathrm{ka} \mathrm{BP}$, Veg. Hist. Archaeobot., 27, 365-379, https://doi.org/10.1007/s00334-017-0653-8, 2018.
Trondman, A.-K., Gaillard, M.-J., Mazier, F., Sugita, S., Fyfe, R., Nielsen, A. B., Twiddle, C., Barratt, P., Birks, H. J. B., Bjune, A. E., Björkman, L., Broström, A., Caseldine, C., David, R., Dodson, J., Dörfler, W., Fischer, E., van Geel, B., Giesecke, T., Hultberg, T., Kalnina, L., Kangur, M., van der Knaap, P., Koff, T., Kuneš, P., Lagerås, P., Latałowa, M., Lechterbeck, J., Leroyer, C., Leydet, M., Lindbladh, M., Marquer, L., Mitchell, F. J. G., Odgaard, B. V., Peglar, S. M., Persoon, T., Poska, A., Rösch, M., Seppä, H., Veski, S., and Wick, L.: Pollenbased quantitative reconstruction of Holocene regional vegetation cover (plant-functional types and land-cover types) in Europe suitable for climate modeling, Glob. Change Biol., 21, 676697, https://doi.org/10.1111/gcb.12737, 2015.

Velichko, A. A., Andreev, A. A., and Klimanov, V. A.: Paleoenvironmental changes in tundra and forest zones of the former USSR during late Pleistocene and Holocene, in: Environmental changes during the last 15000, 1994.

Vipper, P. B.: Pollen profile CHERNOE, Chernoe Lake, Russia, https://doi.org/10.1594/PANGAEA.739109, 2010.

Volkov, I. A. and Arkhipov, S. A.: Quaternary deposits of the Novosibirsk Region, Joint Institute for Geology, Geophysics and Mineralogy, Siberia Branch, USSR Academy of Sciences, Novosibirsk, 1978 (in Russian).

Werner, K., Tarasov, P. E., Andreev, A. A., Müller, S., Kienast, F., Zech, M., Zech, W., and Diekmann, B.: A 12.5-kyr history of vegetation dynamics and mire development with evidence of Younger Dryas larch presence in the Verkhoyansk Mountains, East Siberia, Russia, Boreas, 39, 56-68, https://doi.org/10.1111/j.1502-3885.2009.00116.x, 2010.

Zudin, A. N. and Votakh, M. R.: The stratigraphy of Pliocene and Quaternary strata of Priobskogo Plateau, Nauka, Novosibirsk, 1977 (in Russian). 